\title{
TOWARDS A TRANSPARENT BOUNDARY CONDITION FOR COMPRESSIBLE NAVIER-STOKES EQUATIONS
}

\author{
C.H. BRUNEAU ${ }^{\dagger}$ and E. CREUSE ${ }^{\dagger, \ddagger}$ \\ $\dagger$ Mathématiques Appliquées de Bordeaux, Université Bordeaux 1 F-33405 Talence, France. \\ $\ddagger$ MACS, Université Valenciennes, le Mont Houy F-59304 Valenciennes, France.
}

\begin{abstract}
SUMMARY
A new artificial boundary condition for 2D subsonic flows governed by the compressible Navier-Stokes equations is derived. It is based on the hyperbolic part of the equations, according to the way of propagation of the characteristic waves. A reference flow as well as a convection velocity are used to properly discretize the terms corresponding to the entering waves. Numerical tests on various classical model problems whose solution is known and comparisons with other boundary conditions show the efficiency of the boundary condition. Direct numerical simulations of more complex flows over a dihedral plate are simulated, without creation of acoustic waves going back in the flow.
\end{abstract}

KEY WORDS : Compressible Navier-Stokes equations, Artificial boundary conditions, Subsonic evolution.

\section{INTRODUCTION}

In open flow simulations, the fluid evolves in an infinite domain and thus the computing domain must be obviously truncated. An artificial boundary $\Gamma$ is then introduced, on which appropriate artificial Boundary Conditions (BC) have to be imposed. If the solution obtained on a given truncated domain $\Omega$ is equal to the restriction of the infinite solution on the domain $\Omega$, then the artificial BC is perfect, and called transparent BC. Unfortunately, such a configuration is only an ideal case. A luxurious literature has been dealing with this subject for more than twenty years. We first propose in this introduction to quote some papers, focussing on compressible, subsonic and viscous flows evolutions, governed by the unsteady compressible Navier-Stokes equations.

On one hand, a good BC should lead to a well-posed mathematical problem. Roughly speaking, leading to a stable solution with respect to the initial datum. Following the work of Kreiss for the purely hyperbolic systems [1], Strikwerda established the number and the kind of BCs to impose on an artificial frontier for incompletely parabolic systems in order to ensure well-posedness [2]. The proof relies on several simplifying hypothesis which allow to work on the linearized system with constant coefficients in the half space. With a Fourier-Laplace transformation in space-time, an Ordinary Differential Equation (ODE) is obtained. The number of BCs to impose on a given artificial boundary is then equal to the solutions space dimension of the previous ODE. Non viscous BCs, coming from 
the hyperbolic part of the equation, are distinguished from the viscous BCs, coming from the diffusive part, which have to vanish when the diffusion terms do. This very technical approach is based on the pseudo-differential opertor's theory, but Higdon [3] gives a more simple physical interpretation of it. By using an energy method on the linearized equations, Gustafsson and Sundstrom [4] and Oliger and Sundstrom [5] propose a new BC for artificial boundaries. Dutt [6] works on the non-linearized equations, but with the help of simplifying hypothesis. More recently, Hesthaven and Gottlieb [7] derive an artificial BC to reach a mathematically well-posed problem. In conclusion, if these artificial BCs are mathematically relevant, numerical results are not always satisfying. For instance, Oliger and Sundstrom [5] prove that imposing the pressure on a subsonic compressible outflow leads to a well-posed problem. Unfortunately, such a crude BC gives rise to strong reflections in the flow when hydrodynamic disturbances cross the boundary [8].

On the other hand, another way to define artificial BCs is the concept of non-reflecting $\mathrm{BC}$, which inhibit the reflection of disturbances on the boundary. They are deduced from the compressible Euler equations, considered as a first approximation of the compressible Navier-Stokes equations. In the work of Hedstrom [9] and Thompson [10], the principle is to cancel the wave entering the computational domain. Rudy and Strikwerda [8] adapt the work of Enquist and Majda [11] and Hedstrom [9] to the compressible Navier-Stokes equations, and improve it to find a partially non-reflecting BC. In fact, they give a way to specify the static pressure through a subsonic outflow, which is physically determined by the outside of the computational domain. Other methods exist to specify artificial BCs based on physical arguments, like for example the radiative BC of Bayliss and Turkel, at a subsonic but stationary outflow [12]. More recently, another artificial BC for the compressible Navier-Stokes equation has been developped by Tourette [13],[14], following the work of Halpern [15], [16]. Finally, Thompson derived artificial BCs [17], like the force-free $\mathrm{BC}$, when the non-reflecting $\mathrm{BC}$ is not appropriate. It is the case when the "correct" solution implicitly contains an incoming wave which must not be suppressed. This situation arises when the solution outside the domain is changing in time and its behaviour has to be specified inside the domain through the boundaries.

When the BCs needed for solving the problem are obtained, which are called here physical $\mathrm{BCs}$, it is necessary to adapt them to the numerical implementation and it is also needed to complete them with further BCs, called this time numerical BCs. These extra BCs are not required by the physics of the problem, but only by the resolution algorithm which needs to have the value of all the variables on the boundary. They have to be chosen in a good way. One of the simplest way is to use extrapolations [18], to deduce missing variables by using their values inside the computational domain. Another possibility is to choose numerical BCs consistent with the physical BCs [19],[20],[21].

Nowadays, there are not yet artificial BC for a subsonic compressible outflow which are transparent for all configurations. We shall see that if some of them are successful with the vorticity, they generate in return significant acoustic reflections [22],[23]. These reflected waves can sometimes be responsible for important changes in the behaviour of the flow [24], and even lead to non-physical solutions [25]. To avoid such phenomena, another way is to use a buffer zone, adjacent to the physical zone of interest, in which the solution and/or the equations are gradually modified, so as to reduce disturbances at the outflow boundary. A first type of problems found in the literature using this technique is the boundary layer evolution simulation. For it, Street and Macaraeg [26] and Liu et al [27] 
for incompressible flows, and latter Pruett et al [28] for compressible ones use a parabolization procedure. Wasistho et al [29] bring the flow to a reference one, using a rather crude method. A second type of poblems is to make a vortex leaving the computational domain with as little acoustic reflections as possible. Colonius et al [23] stretch the mesh in the flow direction and filter the solution, making the perturbations less and less resolved, hoping that they will be nearly canceled before interacting with the outflow boundary. This method is efficient, but very costly from the numerical point of view. More recently, following Berenger for electromagnetics [30], Hu [31] and Tam et al [32] apply the perfectly matched layer technique for the linearized Euler equations. This method is discussed by Hesthaven [33]. As explained by Grinstein [24], the usefulness of the buffer approach is restricted to isolating a region of the flow for relatively short timed unsteady simulations, or for simulations that are either forced or mainly focussed on the initial shear flow dynamics. That's why they are not reliable for all configurations.

The object of this paper is to make the artificial frontier as transparent as possible in order to let the flow leaving the computational domain without any perturbation. In particular, the vortices should not create strong reflections. So, our goal is to improve the classical non-reflecting $\mathrm{BC}$, in order to significantly decrease the acoustic reflections. Instead of setting the amplitude of the entering characteristic waves equal to zero, we propose to keep them and discretize them as accurately as possible. As these waves come from outside the domain, we need the values of primitive variables at fictitious points outside the domain to write down the discretization. So, the main work is to find a way to get the best possible values at these fictitious nodes. This is done by using a steady reference flow and a convection reference velocity of the flow at the artificial frontier.

We first recall the compressible Navier-Stokes equations, and the characteritic wave amplitude definition. The numerical approximation used is then precised, and the present BC carefully explained. Classical and representative numerical tests are performed to show its efficiency. Finally, a direct numerical simulation on a dihedral plate is performed.

\section{GOVERNING EQUATIONS}

The governing equations are the 2D compressible Navier-Stokes equations, given in their non-dimensionalized formulation, in an orthonormal system and with usual notations by :

$$
\left\{\begin{aligned}
\frac{\partial U}{\partial t}+\frac{\partial F_{x}(U)}{\partial x}+\frac{\partial F_{y}(U)}{\partial y} & =\frac{1}{R e}\left(\frac{\partial G_{x}(U, \nabla U)}{\partial x}+\frac{\partial G_{y}(U, \nabla U)}{\partial y}\right) \\
U(t=0) & =U_{0}
\end{aligned}\right.
$$

with :

$$
\begin{array}{r}
U=\left[\begin{array}{c}
\rho \\
\rho u \\
\rho v \\
\rho E
\end{array}\right] \quad F_{x}(U)=\left[\begin{array}{l}
\rho u \\
\rho u^{2}+p \\
\rho u v \\
(\rho E+p) u
\end{array}\right] \quad F_{y}(U)=\left[\begin{array}{l}
\rho v \\
\rho u v \\
\rho v^{2}+p \\
(\rho E+p) v
\end{array}\right] \\
G_{x}(U, \nabla U)=\left[\begin{array}{l}
0 \\
\sigma_{x x} \\
\sigma_{x y} \\
\beta_{x}
\end{array}\right] \quad G_{y}(U, \nabla U)=\left[\begin{array}{l}
0 \\
\sigma_{x y} \\
\sigma_{y y} \\
\beta_{y}
\end{array}\right]
\end{array}
$$


where :

$$
\left\{\begin{array} { r l } 
{ \sigma _ { x x } } & { = \frac { 4 } { 3 } \frac { \partial u } { \partial x } - \frac { 2 } { 3 } \frac { \partial v } { \partial y } } \\
{ \sigma _ { y y } } & { = \frac { 4 } { 3 } \frac { \partial v } { \partial y } - \frac { 2 } { 3 } \frac { \partial u } { \partial x } } \\
{ \sigma _ { x y } } & { = \frac { \partial u } { \partial y } + \frac { \partial v } { \partial x } }
\end{array} \quad \left\{\begin{array}{l}
\beta_{x}=u \sigma_{x x}+v \sigma_{x y}+\frac{\gamma k}{\operatorname{Pr}} \frac{\partial T}{\partial x} \\
\beta_{y}=u \sigma_{x y}+v \sigma_{y y}+\frac{\gamma k}{\operatorname{Pr}} \frac{\partial T}{\partial y}
\end{array}\right.\right.
$$

$\gamma, k$ and $\operatorname{Pr}$ are respectively the perfect gaz constant, the thermal conductivity coefficient and the Prandtl number, considered as constant for the simulations we are dealing with. In order to close the system, the two following relations have to be added :

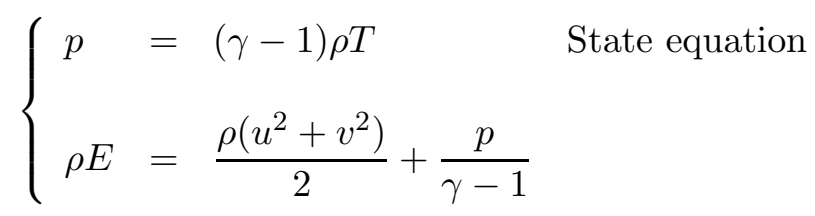

The Reynolds number of the flow, Re, is defined by :

$$
R e=\frac{u_{c} l_{c}}{\nu}
$$

where $u_{c}, l_{c}$ and $\nu$ are respectively a characteristic velocity, a characteristic length and the viscosity of the fluid.

As the domain is bounded, it is necessary to specify some BCs at the frontier. In this work, three BCs are used :

- (1) A subsonic inflow with velocity and temperature imposed :

$$
\left\{\begin{array}{rrr}
u & = & u_{\text {analy }}(x, y) \\
v & = & v_{\text {analy }}(x, y) \\
T & = & T_{0}
\end{array}\right.
$$

- (2) An isothermal no slip wall :

$$
\left\{\begin{array}{l}
u=0 \\
v=0 \\
T=T_{0}
\end{array}\right.
$$

- (3) A subsonic outflow.

BC (1) and BC (2) are precisely described in [19]. The density $\rho$ is computed from the continuity equation on the boundary itself, and the pressure is deduced from the state equation. It should be noted that for the BC (1), only three conditions are used while Strikwerda claims that four conditions are needed for a two-dimensional subsonic inflow [2]. This feature is picked out by Poinsot and Lele, and is coming from the NSCBC method used to derive this subsonic inflow BC [19]. Thus, even if this BC is not in good agreement with the theory, it will be used in this work because of its efficient numerical behaviour in a lot of different configurations [19]. BC (3) is the main object of this work, and is 
carefully described in the next sections.

\section{CHARACTERISTIC WAVES}

In order to simplify the expression, let us consider a node $A$, located on a boundary $x=$ constant, whose outward normal to the boundary is $\vec{n}=(1,0)^{T}$. We reformulate the equations at node $A$ to derive the amplitude of the characteristic waves normal to the boundary.

$$
\begin{aligned}
\frac{\partial U}{\partial t}+\frac{\partial F_{x}(U)}{\partial x}+\frac{\partial F_{y}(U)}{\partial y} \quad & =\frac{1}{R e}\left(\frac{\partial G_{x}(U, \nabla U)}{\partial x}+\frac{\partial G_{y}(U, \nabla U)}{\partial y}\right) \\
\Longleftrightarrow \frac{\partial U}{\partial t}+P P^{-1} \frac{\partial F_{x}(U)}{\partial x}+\frac{\partial F_{y}(U)}{\partial y} & =\frac{1}{R e}\left(\frac{\partial G_{x}(U, \nabla U)}{\partial x}+\frac{\partial G_{y}(U, \nabla U)}{\partial y}\right) \\
\text { with } P=\frac{\partial U}{\partial V} & \text { and } \quad V=[\rho, u, v, p]^{T} \\
\Longleftrightarrow \frac{\partial U}{\partial t}+P P^{-1} Q_{x} \frac{\partial V}{\partial x}+\frac{\partial F_{y}(U)}{\partial y} & =\frac{1}{R e}\left(\frac{\partial G_{x}(U, \nabla U)}{\partial x}+\frac{\partial G_{y}(U, \nabla U)}{\partial y}\right) \\
\qquad \frac{\partial U}{\partial t}+P A_{x} \frac{\partial V}{\partial x}+\frac{\partial F_{y}(U)}{\partial y} & =\frac{1}{R e}\left(\frac{\partial G_{x}(U, \nabla U)}{\partial x}+\frac{\partial G_{y}(U, \nabla U)}{\partial y}\right) \\
\text { with } A_{x}=P^{-1} Q_{x} &
\end{aligned}
$$

$A_{x}$ is a diagonalisable matrix, thanks to the hyperbolic nature of the convective term in the equations. Then, by noting $\Lambda$ the diagonal matrix, and $S$ the transformation matrix whose columns are the $A_{x}$ right eigenvectors,

$$
\begin{array}{cl}
\Longleftrightarrow \frac{\partial U}{\partial t} \quad+P S \Lambda S^{-1} \frac{\partial V}{\partial x}+\frac{\partial F_{y}(U)}{\partial y} & =\frac{1}{R e}\left(\frac{\partial G_{x}(U, \nabla U)}{\partial x}+\frac{\partial G_{y}(U, \nabla U)}{\partial y}\right) \\
\Longleftrightarrow \frac{\partial U}{\partial t}+P S L+\frac{\partial F_{y}(U)}{\partial y} & =\frac{1}{R e}\left(\frac{\partial G_{x}(U, \nabla U)}{\partial x}+\frac{\partial G_{y}(U, \nabla U)}{\partial y}\right) \\
\text { with } L=\Lambda S^{-1} \frac{\partial V}{\partial x} & =S^{-1} P^{-1} \frac{1}{R e}\left(\frac{\partial G_{x}(U, \nabla U)}{\partial x}+\frac{\partial G_{y}(U, \nabla U)}{\partial y}\right)
\end{array}
$$

This last formulation points out the characteristic waves amplitudes vector $L$. By noting $c$ the local sound speed,

$$
c=\sqrt{\frac{\gamma p}{\rho}},
$$

we can show that [19] : 


$$
L=\left[\begin{array}{l}
L_{1} \\
L_{2} \\
L_{3} \\
L_{4}
\end{array}\right]=\left[\begin{array}{c}
(u-c)\left(\frac{\partial p}{\partial x}-\rho c \frac{\partial u}{\partial x}\right) \\
u\left(c^{2} \frac{\partial \rho}{\partial x}-\frac{\partial p}{\partial x}\right) \\
u\left(\frac{\partial v}{\partial x}\right) \\
(u+c)\left(\frac{\partial p}{\partial x}+\rho c \frac{\partial u}{\partial x}\right)
\end{array}\right]=\left[\begin{array}{c}
\lambda_{1}\left(\frac{\partial p}{\partial x}-\rho c \frac{\partial u}{\partial x}\right) \\
\lambda_{2}\left(c^{2} \frac{\partial \rho}{\partial x}-\frac{\partial p}{\partial x}\right) \\
\lambda_{3}\left(\frac{\partial v}{\partial x}\right) \\
\lambda_{4}\left(\frac{\partial p}{\partial x}+\rho c \frac{\partial u}{\partial x}\right)
\end{array}\right]
$$

$L_{k}$ is called the amplitude of the $k^{\text {th }}$ characteristic wave. According to the sign of the eigenvalues, the discretization at the boundary has to be done from inside or outside the domain as we have :

- $\lambda_{k}>0$ means that the wave is leaving the computational domain. In other words, the information carried by the characteristic is going out of the domain.

- $\lambda_{k}<0$ means that the wave is entering the computational domain. In other words, the information carried by the characteristic is coming from outside the domain. As a consequence, a non-viscous physical boundary condition is needed to evaluate $L_{k}$.

\section{NUMERICAL APPROXIMATION}

We perform a Direct Numerical Simulation, using a mixed finite volumes-finite elements method, developped on an unstructured mesh with triangular cells. This section is devoted to briefly explain its implementation for an internal node, that is to say which is not located on a boundary.

\section{$\underline{\text { The convective derivative }}$}

The convective derivative at node $A_{l},\left(\frac{\partial F_{x}}{\partial x}+\frac{\partial F_{y}}{\partial y}\right)_{l}$, is evaluated by a vertex-based finite volumes method associated to Roe solver. Let $C_{l}$ be the control volume whose boundary $\partial C_{l}$ joins the middle of the cells and segments surrounding $A_{l}$, and $\vec{n}_{l}$ be the outward normal to $C_{l}$, we note $\partial C_{l m}=\partial C_{l} \cap \partial C_{m}$ (FIGURE 1$)$ and we have :

$$
\operatorname{area}\left(C_{l}\right)\left(\frac{\partial F_{x}}{\partial x}(U)+\frac{\partial F_{y}}{\partial y}(U)\right)_{l}=\sum_{A_{m} \in K(l)} \Phi_{l m}\left(U_{l}, U_{m}, \overrightarrow{n_{l m}}\right)
$$

with :

and :

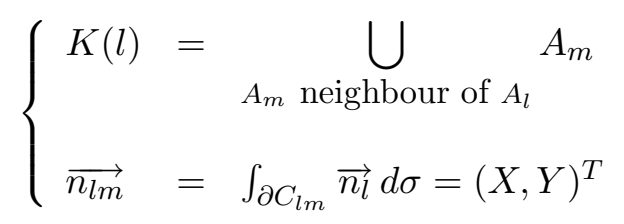

$\Phi_{l m}\left(U_{l}, U_{m}, \overrightarrow{n_{l m}}\right)=\left(X \cdot \frac{F_{x}\left(U_{l}\right)+F_{x}\left(U_{m}\right)}{2}+Y \cdot \frac{F_{y}\left(U_{l}\right)+F_{y}\left(U_{m}\right)}{2}\right)-\frac{1}{2} \sum_{k=1}^{4}\left\|\overline{\overline{\lambda_{k}}}\right\| \quad \delta \omega_{k} \quad \overline{\overline{r_{k}}}$

where $\overline{\overline{r_{k}}}$ is the $k^{\text {th }}$ right eigenvector associated with the $k^{\text {th }}$ eigenvalue $\overline{\overline{\lambda_{k}}}$ of the Roe matrix $\overline{\bar{A}}$, evaluated at the well-known Roe averaged $\overline{\bar{U}}$ (see for instance [34]) function of $U_{l}$ and $U_{m}$ :

$$
\overline{\bar{A}}=X \cdot \frac{\partial F_{x}}{\partial U}(\overline{\bar{U}})+Y \cdot \frac{\partial F_{y}}{\partial U}(\overline{\bar{U}}) .
$$


If we note $\overline{\bar{R}}$ the matrix whose columns are $\overline{\overline{r_{k}}} \quad(1 \leq k \leq 4), \delta \omega_{k}$ is the $k^{\text {th }}$ component of the characteristic variations vector $\delta w$ defined by :

$$
\delta w=(\overline{\bar{R}})^{-1}\left(U_{m}-U_{l}\right)
$$

In order to get the second order accuracy in space, we replace the $U_{l}$ and $U_{m}$ variables in $\Phi_{l m}\left(U_{l}, U_{m}, \overrightarrow{n_{l m}}\right)$ by $U_{l}^{+}$and $U_{m}^{-}$using a MUSCL method :

$$
\begin{aligned}
& U_{l}^{+}=U_{l}+\frac{1}{2} \overrightarrow{\nabla U_{l}} \cdot \overrightarrow{A_{l} A_{m}} \\
& U_{m}^{-}=U_{m}-\frac{1}{2} \overrightarrow{\nabla U_{m}} \cdot \overrightarrow{A_{l} A_{m}}
\end{aligned}
$$

The gradients are evaluated as in [35]. We first define :

$$
\begin{gathered}
(\underline{\overrightarrow{\nabla U}})_{l} \cdot A_{l} A_{m}=(\overrightarrow{\overrightarrow{\nabla U}})_{m} \cdot A_{l} A_{m}=U_{m}-U_{l} \\
(\overrightarrow{\overrightarrow{\nabla U}})_{l}=\frac{1}{\operatorname{area}\left(C_{l}\right)} \sum_{\tau, A_{l} \in \tau} \frac{\operatorname{area}(\tau)}{3} \sum_{k, A_{k} \in \tau} U_{k} \overrightarrow{\nabla \Psi_{k}} \\
(\underline{\overrightarrow{\nabla U}})_{m}=\frac{1}{\operatorname{area}\left(C_{m}\right)} \sum_{\tau, A_{m} \in \tau} \frac{\operatorname{area}(\tau)}{3} \sum_{k, A_{k} \in \tau} U_{k} \overrightarrow{\nabla \Psi_{k}}
\end{gathered}
$$

where $\Psi_{k}$ is the classical linear base function associated to the node $A_{k}$ in a $P_{1}$ finite elements formulation. Then,

$$
\left\{\begin{array}{cc}
\overrightarrow{\nabla U_{l}}= & (1-\beta)(\underline{\overrightarrow{\nabla U}})_{l}+\beta(\overrightarrow{\overrightarrow{\nabla U}})_{l} \\
\overrightarrow{\nabla U_{m}}= & (1-\beta)(\overrightarrow{\overrightarrow{\nabla U}})_{m}+\beta(\underline{\overrightarrow{\nabla U}})_{m} \\
& 0 \leq \beta \leq 1
\end{array}\right.
$$

We choose $\beta=1 / 3$. No flux limiter is needed, because of the smoothness of the solutions we are simulating.

\section{The diffusive derivative}

The diffusive derivative at node $A_{l}, \frac{1}{R e}\left(\frac{\partial G_{x}}{\partial x}+\frac{\partial G_{y}}{\partial y}\right)_{l}$, is evaluated by a classical $P_{1}$ finite elements method. This is a centered process, with the second order accuracy in space. Let $S_{l}$ be the control volume made of the cells $\tau_{m}$ surrounding the node $A_{l}$ and $\overrightarrow{\nu_{l}}$ be the outward normal to $S_{l}$ (FIGURE 1), we have :

$$
\frac{1}{R e}\left(\frac{\partial G_{x}}{\partial x}(U, \nabla U)+\frac{\partial G_{y}}{\partial y}(U, \nabla U)\right)_{l}=\frac{1}{\operatorname{area}\left(S_{l}\right)} \quad \Phi_{l}^{V}=\frac{1}{\operatorname{area}\left(S_{l}\right)} \sum_{\tau_{m}, A_{l} \in \tau_{m}} \Phi_{\tau_{m}, l}^{V}
$$

with :

$$
\Phi_{\tau_{m}, l}^{V}=-\frac{1}{R e} \operatorname{area}\left(\tau_{m}\right)\left(G_{x}(U, \nabla U) \frac{\partial \Psi_{l}}{\partial x}+G_{y}(U, \nabla U) \frac{\partial \Psi_{l}}{\partial y}\right)
$$

where $\frac{\partial \Psi_{l}}{\partial x}$ (resp. $\frac{\partial \Psi_{l}}{\partial y}$ ) is the derivative in $x$ (resp. $y$ ) inside $\tau_{m}$ of the base function associated to the node $A_{l} ; G_{x}(U, \nabla U)$ and $G_{y}(U, \nabla U)$ are constant quantities inside $\tau_{m}$, 
provided $u$ and $v$ are defined in $\tau_{m}$ by :

$$
\left\{\begin{array}{l}
u_{m o y}^{\tau_{m}}=\frac{1}{3} \sum_{k, A_{k} \in \tau_{m}} u_{k} \\
v_{m o y}^{\tau_{m}}=\frac{1}{3} \sum_{k, A_{k} \in \tau_{m}} v_{k} .
\end{array}\right.
$$

The integration in time

We denote $U_{l}^{n}$ the solution at node $A_{l}$ and at time $t^{n}$. In order to compute $U_{l}^{n+1}$, we use the Henn method to bring second order accuracy in time. We first begin to define the full residual at node $A_{l}$ and at time $t^{n}$ :

$$
R\left(U_{l}^{n}\right)=R_{l}^{n}=\left(\Phi_{l}^{V}\right)^{n}-\sum_{A_{m} \in K(l)} \Phi_{l m}^{n}\left(U_{l}^{n}, U_{m}^{n}, \overrightarrow{n_{l m}}\right)
$$

and the resolution of the ODE :

$$
\operatorname{area}\left(C_{l}\right)\left(\frac{\partial U}{\partial t}\right)_{l}=R_{l}^{n}
$$

gives the value $U_{l}^{n+1}$ at time $t^{n+1}=t^{n}+\Delta t$ by :

$$
\left\{\begin{array}{l}
U_{l}^{*}=U_{l}^{n}+\frac{\Delta t}{\operatorname{area}\left(C_{l}\right)} R_{l}^{n} \\
U_{l}^{n+1}=U_{l}^{n}+\frac{\Delta t}{\operatorname{area}\left(C_{l}\right)}\left(\frac{R_{l}^{n}+R_{l}^{*}}{2}\right)
\end{array}\right.
$$

where $R_{l}^{*}=R\left(U_{l}^{*}\right)$.

Remark : The way to proceed is justified because of the mass-lumping approximation, which can be written :

$$
\sum_{A_{m}} U_{m} \int_{S_{l}} \Psi_{l} \Psi_{m} d S \approx U_{l} \int_{S_{l}} \sum_{A_{m}} \Psi_{m} \Psi_{l} d S=U_{l} \int_{S_{l}} \Psi_{l} d S
$$

Moreover, as the scheme is explicit, a CFL condition needs to be imposed [36].

\section{THE PRESENT ARTIFICIAL BOUNDARY CONDITION}

In order to simplify the explanation, we assume here that the mesh is an uniform cartesian mesh, and note $A_{i, j}$ the current point on the outflow boundary $\Gamma$ at $x=$ constant (FIGURE 2 ). As a consequence, the previous subscript $l$ is replaced by the couple of subscripts $(i, j)$.

- The tangential convective derivative at node $A_{i, j}$ and at time $t^{n},\left(\frac{\partial F_{y}}{\partial y}\right)_{i, j}^{n}$, is computed in the same way than inside the computational domain, but degenerates to one dimension. Here, the estimation of $\left(\frac{\partial F_{y}}{\partial y}\right)_{i, j}^{n}$ is evaluated with a 1D Roe scheme and a MUSCL technique, and the control volume $C_{i, j}$ becomes a segment on the boundary whose middle is $A_{i, j}$. 
- The diffusive residual $\left(\Phi_{i, j}^{V}\right)^{n}$ is computed in the same way than inside the computational domain. More precisely, the control volume $S_{i, j}$ is always made of the cells surrounding $A_{i, j}$. Moreover, a boundary term needs to be evaluated whose expression is :

$$
\frac{1}{R e} \int_{\partial S_{i, j} \cap \Gamma} G_{x}(U, \nabla U) \Psi_{i, j} d \sigma .
$$

Viscous boundary conditions are then imposed. Since these additional conditions must have a negligible effect when the viscosity goes to zero, they are only used to modify the conservation equations before to discretize them at the subsonic outflow boundary nodes [19]. They are given by :

$$
\left\{\begin{array}{l}
\frac{\partial^{2} T}{\partial x^{2}}=0 \\
\frac{\partial \sigma_{x y}}{\partial x}=0
\end{array}\right.
$$

The normal convective derivative $\left(\frac{\partial F_{x}}{\partial x}\right)_{i, j}^{n}$ is completely defined by the evaluation of $(L)_{i, j}^{n}$.

- If $\left(\lambda_{k}\right)_{i, j}^{n}>0$, then the characteristic curve is leaving the computational domain. As a matter on fact, $\left(L_{k}\right)_{i, j}^{n}$ is approximated from the solution inside the domain. For an outflow subsonic boundary, this is the case for $\left(L_{2}\right)_{i, j}^{n},\left(L_{3}\right)_{i, j}^{n}$ and $\left(L_{4}\right)_{i, j}^{n}$. The discretisation is then performed with a second order scheme as :

$$
\left\{\begin{array}{l}
\left(L_{2}\right)_{i, j}^{n}=u_{i, j}^{n}\left(\left(c_{i, j}^{n}\right)^{2} \frac{\rho_{i-2, j}^{n}-4 \rho_{i-1, j}^{n}+3 \rho_{i, j}^{n}}{2 \Delta x}-\frac{p_{i-2, j}^{n}-4 p_{i-1, j}^{n}+3 p_{i, j}^{n}}{2 \Delta x}\right) \\
\left(L_{3}\right)_{i, j}^{n}=u_{i, j}^{n}\left(\frac{v_{i-2, j}^{n}-4 v_{i-1, j}^{n}+3 v_{i, j}^{n}}{2 \Delta x}\right) \\
\left(L_{4}\right)_{i, j}^{n}=\left(u_{i, j}^{n}+c_{i, j}^{n}\right)\left(\frac{p_{i-2, j}^{n}-4 p_{i-1, j}^{n}+3 p_{i, j}^{n}}{2 \Delta x}+\rho_{i, j}^{n} c_{i, j}^{n} \frac{u_{i-2, j}^{n}-4 u_{i-1, j}^{n}+3 u_{i, j}^{n}}{2 \Delta x}\right)
\end{array}\right.
$$

- If $\left(\lambda_{k}\right)_{i, j}^{n}<0$, then the characteristic is coming from outside the computational domain. Now, we can not approximate $\left(L_{k}\right)_{i, j}^{n}$ from the solution inside the domain because it would generate instabilities. Extrapolation methods are also inadapted. For instance, a first order extrapolation on $p$ and $u$ in order to discretise $\left(L_{k}\right)_{i, j}^{n}$ would lead to the same gradients as inside the domain, what is unstable. For an outflow subsonic boundary, this is the case for $\left(L_{1}\right)_{i, j}^{n}$.

A first way to proceed is the Non-Reflecting Boundary Condition (NRBC) (see for instance [19]):

$$
\begin{array}{ll}
\left(L_{1}\right)_{i, j}^{n}=0 & \text { for a subsonic outflow ; } \\
\left(L_{1}\right)_{i, j}^{n}=\left(L_{2}\right)_{i, j}^{n}=\left(L_{3}\right)_{i, j}^{n}=0 & \text { for a subsonic inflow. }
\end{array}
$$

Nevertheless, a pressure drift can arise because the static pressure at infinity $p_{\infty}$ is not specified to the flow. In order to avoid such a drawback, a pressure recall can be added to obtain the Partially Non-Reflecting Boundary Condition (PNRBC) :

$$
\left(L_{1}\right)_{i, j}^{n}=\frac{\sigma\left(1-M^{2}\right) c_{i, j}^{n}\left(p_{i, j}^{n}-p_{\infty}\right)}{l}
$$


with :

$$
\begin{cases}\sigma & : \text { the reflection parameter } \\ M & : \text { the maximum Mach number in the flow } \\ c_{i, j}^{n} & : \text { the local speed of sound } \\ l & : \text { the computational domain characteristic length }\end{cases}
$$

The $\sigma$ coefficient needs to be adjusted [8]. As we shall see it, for several numerical tests these BCs do not give good results. Indeed, if the hydrodynamic disturbances are often well evacuated, strong acoustic reflections arise.

We now propose a new method to evaluate $\left(L_{1}\right)_{i, j}^{n}$. Let $A_{i+1, j}$ (resp. $A_{i+2, j}$ ) be a fictitious node, outside the domain, symmetric to $A_{i-1, j}\left(\right.$ resp. $\left.A_{i-2, j}\right)$ with regards to $\Gamma$. If $U_{i+1, j}^{n}$ and $U_{i+2, j}^{n}$ were available, then we would write :

$$
\left(L_{1}\right)_{i, j}^{n}=\left(u_{i, j}^{n}-c_{i, j}^{n}\right)\left(\frac{-p_{i+2, j}^{n}+4 p_{i+1, j}^{n}-3 p_{i, j}^{n}}{2 \Delta x}-\rho_{i, j}^{n} c_{i, j}^{n} \frac{-u_{i+2, j}^{n}+4 u_{i+1, j}^{n}-3 u_{i, j}^{n}}{2 \Delta x}\right)
$$

Nevertheless, the $p_{i+2, j}^{n}, p_{i+1, j}^{n}, u_{i+2, j}^{n}$ and $u_{i+1, j}^{n}$ values are not known, and of course, these exact values are unreachable. The goal of this section is to find a way to approximate them, in order to derive a physically relevant value of $\left(L_{1}\right)_{i, j}^{n}$ which does not produce strong acoustic waves.

1. We first determine a global reference steady state $\bar{V}$, in the neighbourhood of $\Gamma$ :

$$
\bar{V}=\left[\begin{array}{c}
\bar{\rho} \\
\bar{u} \\
\bar{v} \\
\bar{p}
\end{array}\right]
$$

$\bar{V}$ depends on the flow under consideration, and can be obtained either analytically or numerically.

2. Then, $\left(V^{\prime}\right)^{n}$ is defined in the computational domain as a perturbation of $\bar{V}$ :

$$
\left(V^{\prime}\right)^{n}=V^{n}-\bar{V}
$$

and we write :

$$
\begin{aligned}
\left(L_{1}\right)_{i, j}^{n} & =\left(u_{i, j}^{n}-c_{i, j}^{n}\right)\left(\frac{-\bar{p}_{i+2, j}+4 \bar{p}_{i+1, j}-3 \bar{p}_{i, j}}{2 \Delta x}-\rho_{i, j}^{n} c_{i, j}^{n} \frac{-\bar{u}_{i+2, j}+4 \bar{u}_{i+1, j}-3 \bar{u}_{i, j}}{2 \Delta x}\right) \\
& +\left(u_{i, j}^{n}-c_{i, j}^{n}\right)\left(\frac{-\left(p^{\prime}\right)_{i+2, j}^{n}+4\left(p^{\prime}\right)_{i+1, j}^{n}-3\left(p^{\prime}\right)_{i, j}^{n}}{2 \Delta x}-\rho_{i, j}^{n} c_{i, j}^{n} \frac{-\left(u^{\prime}\right)_{i+2, j}^{n}+4\left(u^{\prime}\right)_{i+1, j}^{n}-3\left(u^{\prime}\right)_{i, j}^{n}}{2 \Delta x}\right) \\
& =\left(\overline{L_{1}}\right)_{i, j}^{n}+\left(L_{1}^{\prime}\right)_{i, j}^{n}
\end{aligned}
$$

$\left(\overline{L_{1}}\right)_{i, j}^{n}$ is easily computed with the knowledge of $\bar{V}$.

3. We assume that the perturbations around $\bar{V}$ at node $A_{i, j}$ are convected with a convection velocity $u_{\text {conv }}$, which depends on the flow under consideration as we shall see later. Mainly, this convection velocity is the mean flow velocity. Then, we make the approximation :

$$
\left(L_{1}^{\prime}\right)_{i, j}^{n} \approx\left(u_{i, j}^{n}-c_{i, j}^{n}\right)\left(\frac{-\left(p^{\prime}\right)_{i, j}^{n-2 \alpha}+4\left(p^{\prime}\right)_{i, j}^{n-\alpha}-3\left(p^{\prime}\right)_{i, j}^{n}}{2 \Delta x}-\rho_{i, j}^{n} c_{i, j}^{n} \frac{-\left(u^{\prime}\right)_{i, j}^{n-2 \alpha}+4\left(u^{\prime}\right)_{i, j}^{n-\alpha}-3\left(u^{\prime}\right)_{i, j}^{n}}{2 \Delta x}\right)
$$


with $t_{n-\alpha}=t_{n}-\alpha \Delta t$ and $\alpha=\frac{\Delta x}{u_{\text {conv }} \Delta t}$. Since $\bar{V}$ is a steady state, we have :

$$
\left(L_{1}^{\prime}\right)_{i, j}^{n} \approx\left(u_{i, j}^{n}-c_{i, j}^{n}\right)\left(\frac{-p_{i, j}^{n-2 \alpha}+4 p_{i, j}^{n-\alpha}-3 p_{i, j}^{n}}{2 \Delta x}-\rho_{i, j}^{n} c_{i, j}^{n} \frac{-u_{i, j}^{n-2 \alpha}+4 u_{i, j}^{n-\alpha}-3 u_{i, j}^{n}}{2 \Delta x}\right)
$$

The methodology presented above can easily be adapted to other approximations following the same way to give values of the necessary quantities at the fictitious nodes. We point out to the reader that $u_{\text {conv }}$ can not be equal to zero.

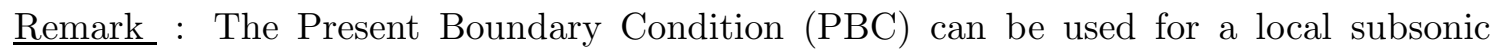
inflow. In this particular case, $u_{i, j}^{n} \leq 0$, and $\left(L_{2}\right)_{i, j}^{n}$ and $\left(L_{3}\right)_{i, j}^{n}$ have also to be specified and can not be approximated from the solution inside the domain. In fact, the way to proceed is exactly the same than for $\left(L_{1}\right)_{i, j}^{n}$, and we would have:

$$
\begin{aligned}
& \left(L_{2}\right)_{i, j}^{n}=\left(\overline{L_{2}}\right)_{i, j}^{n}+\left(L_{2}^{\prime}\right)_{i, j}^{n} \\
& \left(L_{3}\right)_{i, j}^{n}=\left(\overline{L_{3}}\right)_{i, j}^{n}+\left(L_{3}^{\prime}\right)_{i, j}^{n}
\end{aligned}
$$

\section{CLASSICAL NUMERICAL TESTS}

\subsection{The non-viscous vortex leaving the computational domain}

A first relevant test is the non-viscous vortex leaving the computational domain. An inviscid vortex is superimposed on a simple uniform translation flow. The vortex is defined by its center, and the two velocity components $u_{r}$ and $u_{\theta}$, expressed in the polar coordinates by :

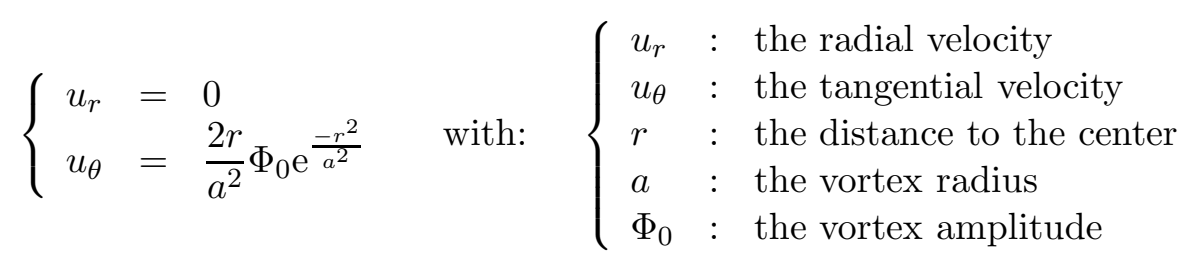

In order to entirely define the initialisation field, the pressure and the density have to be consistent with the velocity. Indeed, imposing a constant pressure would not be satisfying, because acoustic waves would leave the vortex core. In order to do it, pressure and density fields are defined by the following system, which has to be integrated :

$$
\left\{\begin{aligned}
\rho \frac{u_{\theta}^{2}}{r} & =\frac{\partial p}{\partial r} \\
\frac{p}{\rho^{\gamma}} & =\frac{p_{\infty}}{\rho_{\infty}^{\gamma}}
\end{aligned}\right.
$$

A very similar test is presented in several recent papers. Poinsot and Lele [19] first, with a weakly supersonic main flow and a relative maximum velocity in the vortex of $0.18 \%$ of the main flow convective velocity. Then, with a subsonic flow in Colonius et al [23], who define a reflection coefficient by

$$
r(t)=\frac{\|\operatorname{div}(t)\|_{\infty}}{\left\|\omega_{0}\right\|_{\infty}}
$$


where $\|\operatorname{div}(t)\|_{\infty}$ is the $L_{\infty}$ norm of the divergence field at time $t$ and $\left\|\omega_{0}\right\|_{\infty}$ is the $L_{\infty}$ norm of the initial vorticity field. Finally, two initialisations are presented by Nicoud [22] in a subsonic context. The former with a relative maximum velocity in the vortex in the order of $1 \%$ of the main flow velocity, the latter with a relative maximum velocity in the vortex in the order of $90 \%$ of the main flow velocity. He compares the results obtained with a reference solution, deduced either by the analytical resolution of the equations when the amplitude is weak and allows the linearization, or by the same simulation on a longer domain to avoid interaction between the vortex and the outflow boundary. We chose here to present our results like Nicoud did.

The computational domain is a square of side $l=1$. The mesh contains 101 nodes in each direction of space, and is made of 20000 triangular cells. The vortex is initially located at the center of the domain, and on the (a),(b) and (c) boundaries (FIGURE 3), the NRBC is imposed. The vortex radius is $a=0.075 \sqrt{2}$, and the Mach number is $M=0.2$. The main flow convection velocity is $u_{\infty}=1$. The simulation is performed up to $t=1.5$. The PBC and the NRBC are successively applied on the boundary (d). The two solutions are compared to a Reference Solution (RS) obtained by a simulation on a longer domain, to avoid that the vortex interacs with the outflow boundary. The first vortex amplitude, $\Phi_{1}=-0.1105$, leads to a strong vortex $V_{1}$, with a relative maximum velocity in the vortex in the order of $90 \%$ of the main flow velocity. During the evolution, the boundary (d) is an outflow subsonic boundary. The second one, $\Phi_{2}=-0.2210$, leads to a very strong vortex $V_{2}$, with a relative maximum velocity in the vortex in the order of $180 \%$ of the main flow velocity. As a consequence, the global horizontal velocity can become negative during the evolution, and the boundary (d) will have sometimes to treat local inflows.

The reference flow chosen to apply the PBC is here very simple, as it is taken equal to the flow at infinity:

$$
\bar{V}=\left[\rho_{\infty}, u_{\infty}, 0, p_{\infty}\right]^{T} \quad \frac{\partial \bar{V}}{\partial x}=[0,0,0,0]^{T}
$$

The reference convection velocity $u_{c o n v}$ is the velocity at infinity :

$$
u_{\text {conv }}=u_{\infty},
$$

as the vortex is convected at this velocity. Whatever the vortex amplitude is, we can see that the vorticity is well evacuated from the computational domain even if the $\mathrm{PBC}$ solution is closer to the RS solution than the NRBC solution (FIGURE 4). However, the $\mathrm{NRBC}$ gives rise to a reflection coefficient of the order of $2 \%$, whereas the $\mathrm{PBC}$ induces a reflection coefficient in the same order as the one obtained with the RS, namely 0.05 $\%$ (FIGURE 5). This value is due to the numerical discretisation, and can be considered as the smallest reflection coefficient we can get. Finally, qualitatively speaking, at time $t=0.5$, when the vortex center is exactly on the outflow boundary, the vorticity field is a bit perturbed and the pressure field strongly altered by the NRBC, whereas the PBC gives a pressure field nearly identical to the RS. This phenomenon occurs for both vortices $V_{1}$ and for $V_{2}$, and is displayed for $V_{1}$ on Figure 6 .

$\underline{\text { Remark }}$ : If we take a weak vortex with for instance $\Phi_{0}=-0.005$, then the NRBC provides results as good as the $\mathrm{PBC}$ as far as the vorticity field is concerned. Nevertheless, the acoustic field is also altered, like for stronger vortices. 


\subsection{The Poiseuille flow}

A second relevant flow is the Poiseuille flow. It is a steady viscous flow, that allows to test the PBC efficiency in such a configuration. The computational domain is a rectangle, with lenght $L=10$ and half height $l=1$ (FIGURE 7). The mesh contains 101 nodes in the horizontal direction and 21 in the vertical direction, it is then made of 4000 triangular cells. The Mach number is $M=0.1$, and the Reynolds number is $R e=15$.

(a) is a subsonic inflow boundary, with velocity and temperature imposed :

$$
u(0, y)=u_{0}\left[1,0-\left(\frac{y}{l}\right)^{2}\right] \quad ; \quad v(0, y)=0 \quad ; \quad T(0, y)=T_{0}
$$

(b) and (c) are isothermal no-slip walls :

$$
u(x, y)=0 \quad ; \quad v(x, y)=0 \quad ; \quad T(x, y)=T_{0}
$$

The initialisation is :

$$
\rho(x, y)=\rho_{0} \quad ; \quad u(x, y)=0 \quad ; \quad v(x, y)=0 \quad ; \quad p(x, y)=p_{0}
$$

When the pressure difference between the inflow boundary and the outflow boundary is sufficiently small compared to the averaged pressure in the flow, the incompressible model can be considered as a good approximation of the compressible model, so an analytical solution can be deduced to compare our results with. This is the case in our configuration, and when the steady state is reached, the analytical solution is given by :

$$
u_{\text {analy }}(x, y)=u_{0}\left[1,0-\left(\frac{y}{l}\right)^{2}\right] \quad ; \quad v_{\text {analy }}(x, y)=0 \quad ; \quad\left(\frac{\partial p}{\partial x}\right)_{\text {analy }}=-\frac{8 \rho_{0} u_{0}^{2}}{3 \operatorname{Rel}}
$$

The PBC is tested on (d), and compared to the NRBC and the PNRBC with $\sigma=0.5$. The reference flow chosen to apply the PBC is here defined by:

$$
(\bar{V})_{i, j}=\left[\rho_{0},\left(u_{\text {analy }}\right)_{i, j}, 0, p_{0}\right]^{T} \quad\left(\frac{\partial \bar{V}}{\partial x}\right)_{i, j}=\left[0,0,0,\left(\frac{\partial p}{\partial x}\right)_{\text {analy }}\right]^{T}
$$

The convection velocity $u_{c o n v}$ is taken equal to the average value of the velocity profile imposed on the inflow boundary :

$$
u_{\text {conv }}=\frac{2 u_{0}}{3}
$$

The mass flux evolution through the outflow boundary as a function of the time is first analysed, in order to underline the convergence. In fact, The NRBC and the PBC prevent the solution from converging towards a steady state. The static pressure, carried by the entering characteristic, can not be specified to the flow, and pressure drift arises. Nevertheless, this pressure drift is far slower for the PBC than for the NRBC. Indeed, the line slope relative to the $\mathrm{PBC}$ is in the order of 60 times less than the line slope relative to the NRBC. On the other hand, the PNRBC allows the convergence. We then introduce the NRBC-b (resp. PBC-b) which is similar to the NRBC (resp. PBC), but implemented in a modified way : the density and the pressure are strongly specified at nodes $I$ and $J$, so that the convergence is ensured because the static pressure is specified. As we can see (FIGURE 8), the velocity convergence towards the steady state is faster for the PBC-b than for the NRBC-b. Finally, the results are compared between the PNRBC, the NRBC-b, 
and the PBC-b when the steady state is reached. The horizontal velocity profiles are well evaluated, whatever the BC is (FIGURE 9-(a)). The longitudinal pressure gradient is always satisfactory, but the $\mathrm{PBC}-\mathrm{b}$ provides a pressure value at the end of the computation domain closer to the pressure value crudely imposed in nodes $I$ and $J$ than the PNRBC and the NRBC-b (FIGURE 9-(b)), which is a more physical behaviour since the pressure must be independant of $y$.

\subsection{The boundary layer}

The third flow we want to simulate is a boundary layer evolution on a flat plate (FIGURE 10).

The leading edge is located in $\mathrm{O}$, and the computational domain is represented by the dashed line. For each point inside it, the reduced distance is defined as :

$$
\eta=\frac{y}{x+x_{0}} \sqrt{R_{x+x_{0}}}
$$

with :

$$
R_{x+x_{0}}=\frac{u_{\infty}\left(x+x_{0}\right)}{\nu} .
$$

where $\nu$ is the cinematic viscosity of the fluid, and $u_{\infty}$ the horizontal component of the velocity very far from the plate. The theoritical velocity field can then also be deduced, thanks to the resolution of the Blasius equations for incompressible flows. So we can take as a first approximation for a laminar evolution :

$$
\left\{\begin{aligned}
\rho & =\rho_{\infty} \\
u & =u_{\infty} u_{\text {analy }}(\eta) \\
v & =\frac{u_{\infty}}{\sqrt{R_{x+x_{0}}}} v_{\text {analy }}(\eta) \\
p & =p_{\infty}
\end{aligned}\right.
$$

with :

$$
\left\{\begin{array}{l}
u_{\text {analy }}(\eta)=f^{\prime}(\eta) \\
v_{\text {analy }}(\eta)=\frac{1}{2}\left(\eta f^{\prime}(\eta)-f(\eta)\right)
\end{array}\right.
$$

where $f$ and $f^{\prime}$ are tabulated functions of $\eta$ [39].

At abscissa $x+x_{0}$, the thickness of the boundary layer is approximated by :

$$
\delta_{x+x_{0}} \approx \frac{5 \sqrt{x+x_{0}} \sqrt{\nu}}{\sqrt{u_{\infty}}} .
$$

A characteristic Reynolds number of the flow can be defined as :

$$
\operatorname{Re}_{\delta}=\frac{\delta u_{\infty}}{\nu} .
$$

The parameters used in the simulation are :

$$
\begin{aligned}
x_{0} & =5.56 \delta, \text { that is to say } R e_{\delta}=139 \\
L & =\frac{50 \delta}{3} \\
h & =\frac{10 \delta}{3}
\end{aligned}
$$


The mesh is uniform with 61 nodes vertically and 51 nodes horizontally.

The (a) boundary is a subsonic inflow with velocity and temperature imposed :

$$
u(0, y)=u_{\infty} u_{\text {analy }}(0, y), \quad ; \quad v(0, y)=\frac{u_{\infty}}{\sqrt{R_{x_{0}}}} v_{\text {analy }}(\eta) \quad ; \quad T(0, y)=T_{0}
$$

The (b) boundary is an isothermal no-slip wall :

$$
u(x, 0)=0 \quad ; \quad v(x, 0)=0 \quad ; \quad T(x, 0)=T_{0}
$$

The (c) boundary is a PNRBC with $\sigma=0.5$.

The reference flow chosen for the PBC is :

$$
\left\{\begin{array} { l } 
{ \overline { \rho } = \rho _ { \infty } } \\
{ \overline { u } = u _ { \infty } u _ { \text { analy } } ( \eta ) } \\
{ \overline { v } = \frac { u _ { \infty } } { \sqrt { R _ { x _ { 0 } + L } } } v _ { \text { analy } } ( \eta ) } \\
{ \overline { p } = p _ { \infty } }
\end{array} \quad \left\{\begin{array}{l}
\frac{\partial \bar{\rho}}{\partial x}=0 \\
\frac{\partial \bar{u}}{\partial x}=\frac{-u_{\infty} y \sqrt{R_{L+x_{0}}}}{2\left(L+x_{0}\right)^{2}} f^{\prime \prime}(\eta) \\
\frac{\partial \bar{v}}{\partial x}=\frac{-u_{\infty}}{4\left(L+x_{0}\right)}\left(\frac{1}{\sqrt{R_{L+x_{0}}}}\left(\eta f^{\prime}(\eta)-f(\eta)\right)+\frac{\eta f^{\prime \prime}(\eta) y}{\left(L+x_{0}\right)}\right) \\
\frac{\partial \bar{p}}{\partial x}=0
\end{array}\right.\right.
$$

The convection velocity $u_{\text {conv }}$ is equal to the velocity very far from the plate :

$$
u_{\text {conv }}=u_{\infty} .
$$

The PBC is tested on the (d) boundary and compared to the NRBC. The initialisation is given by :

$$
\rho=\rho_{\infty} \quad ; \quad u=v=0 \quad ; \quad p=p_{\infty}
$$

The solution is analysed when the steady state is reached, for which $u_{\text {num }}$ and $v_{\text {num }}$ are defined by :

$$
\left\{\begin{array}{l}
u_{\text {num }}=\frac{u}{u_{\infty}} \\
v_{\text {num }}=\frac{v \sqrt{R_{x+x_{0}}}}{u_{\infty}}
\end{array}\right.
$$

For each BC, $\eta=f\left(u_{\text {num }}\right)$ and $\eta=f\left(v_{\text {num }}\right)$ are respectively compared to $\eta=f\left(u_{\text {analy }}\right)$ and $\eta=f\left(v_{\text {analy }}\right)$, at the middle transverse section $x=\frac{25 \delta}{3}$, and at the outflow transverse section $x=\frac{50 \delta}{3}$ (FIGURE 11$)$.

As we can see, the PBC provides far better results than the NRBC, especially for the vertical velocity profile. The PNRBC can give better results than the NRBC if the $\sigma$ coefficient is well adjusted.

\subsection{The Poiseuille flow with a sound source}

Up to now, the PBC has been applied for vortex dominated flows, or for the computation and analysis of steady state solutions. Even if it is not the topic of this paper, it would be interesting to make a numerical experiment involving a problem dominated by 
sound waves, to show the PBC behaviour for such an aeroacoustic test.

We so consider the established Poiseuille flow on the computational domain defined by a rectangle with lenght $L=1$ and half height $l=1$. The mesh contains 101 nodes in the horizontal direction and 201 in the vertical one; it is then made of 40000 triangular cells. The Mach number is $M=0.1$ and the Reynolds number is $R e=15$. At time $t=0$, an acoustic perturbation is superimposed in the flow, by replacing the pressure and density values $p$ and $\rho$ in all the computational domain by $p^{\prime}$ and $\rho^{\prime}$ defined as :

$$
\left\{\begin{array}{l}
\rho^{\prime}=\rho+\varepsilon \mathrm{e}^{-\frac{\left(x-x_{0}\right)^{2}+\left(y-y_{0}\right)^{2}}{a^{2}}} \\
p^{\prime}=\left(\frac{\rho^{\prime}}{\rho_{\infty}}\right)^{\gamma} p_{\infty}
\end{array}\right.
$$

$\rho_{\infty}$ and $p_{\infty}$ are respectively the static density and the static pressure at infinity. The parameters used in the simulation are $\varepsilon=0.2, x_{0}=0.8, y_{0}=1.0$ and $a=0.05$.

Then, we compare the temporal evolution of the pressure perturbation when the NRBC is used and when the PBC is used, to a reference solution obtained on a longer computational domain to avoid any interaction with the outflow boundary. There is no need to strongly impose the pressure and the density somewhere in the flow like we did in section 6.2 , because of the shortness of the simulation which prevents the flow from any pressure drift. Moreover, such an initialisation provides perturbations on the inflow and horizontal boundaries. Nevertheless, the results are analysed in the window $\{(x, y) ; 0.6 \leq x \leq 1$ and $0.6 \leq y \leq 1.4\}$, so that these perturbations don't have enough time to contaminate the flow in the window.

As we can see for this strong acoustic test case (FIGURE 12), none of the two BCs gives the same isolines than the Reference Solution at time $t=0.04$. Nevertheless, the isolines distribution seems better for the PBC than for the NRBC. If we take now $u_{\text {conv }}=\frac{2 u_{0}}{3}\left(1+\frac{1}{M}\right)$, which is closer to the theoritical velocity of the acoustic wave than the previous $u_{\text {conv }}$, the results are improved. All these observations are confirmed by the comparison of the longitudinal pressure evolution along the axis $y=1$ at time $t=0.04$ (FIGURE 13). Indeed, the concavity of the profiles are respected by the PBC, and the PBC with $u_{\text {conv }}=\frac{2 u_{0}}{3}\left(1+\frac{1}{M}\right)$ gives clearly the closest profile to the reference one. Finally, we conclude that the PBC gives better results than the NRBC on this test. The closer $u_{c o n v}$ is to the velocity of the phenomena inside the computational domain, the better the results are. Thus, it appears that even if the results are less good than for the purely hydrodynamic test cases above, the PBC can be applied successfully to some aeroacoustic test cases.

\section{NUMERICAL SIMULATION ON A PLAN DIHEDRAL}

\subsection{Presentation}

The computational domain is displayed on FIGURE 14. Like for the boundary layer test, $\delta$ is the height of the boundary layer at point A on the inflow boundary (a), and $\delta_{200}$ its value for $R e_{\delta}=200$. The leading edge $\mathrm{O}$ is located at $x_{0}=8 \delta_{200}$ in front of $\mathrm{A}$. We get $\alpha=10^{\circ}$. (b) is a wall, and (c) and (d) are artificial boundaries. The $\mathrm{m}$ and $\mathrm{n}$ parameters 
will be fixed later, and allow to give the dimensions of the computational domain. $\delta$ is deduced from $R e_{\delta}$ with the relation :

$$
\delta=\frac{200}{R e_{\delta}} \delta_{200}
$$

The non-dimensionalized variables are obtained by choosing $l_{c}=\delta_{200}$ and $u_{c}=u_{\infty}$, so that the numerical Reynolds number used for the equations resolution is $R e=\frac{l_{c} u_{c}}{\nu}=\frac{R e_{\delta}^{2}}{200}$. The (a) boundary is a subsonic inflow with velocity and temperature imposed, similarly to the boundary layer test. The (b) boundary is an isothermal no-slip wall. On the (c) boundary, the PNRBC is applied : $\left(L_{1}\right)_{i, j}^{n}=0.1\left(p_{i, j}^{n}-p_{\infty}\right)$.

The mesh is a structured non uniform one. Geometrical progressions are used in order to refine it in the boundary layer and around B, the point of discontinuity on the wall, both vertically and horizontally as shown on (FIGURE 15). It should be noted that for the higher Reynolds number involved in the following simulations, 11 nodes are still present in the boundary layer's height to ensure a sufficiently accurate spatial resolution.

\subsection{The reference solution}

We take here $R e_{\delta}=50, \mathrm{~m}=101$ and $\mathrm{n}=72$. In order to obtain a solution of the equations, a buffer zone adjacent to the computational domain in used. Inside this buffer, the mesh is stretched in the horizontal direction [23], and the solution progressively brought back to a blasius flow along the dihedral [29], which can be considered as a local solution. Consequently, no BC is needed on the (d) boundary. The simulation converges towards a steady state, which is denoted $V_{50}$. No recirculation area, defined by a zone in which horizontal velocities are negative, is noticed. Horizontal velocity and pressure isolines are displayed above the plan dihedral (FIGURE 16). The solution remains the same if we take $\mathrm{m}=150$ instead of $\mathrm{m}=101$, and also if the buffer zone size is twice longer. For higher Reynolds numbers, we can take smaller values of $m$ as the boundary layer becomes thiner.

\subsection{The present $\mathrm{BC}$}

The buffer zone is then removed. The initialisation is made with $V_{50}$, and two simulations are performed at $R e_{\delta}=50$. The former with the NRBC on (d), the latter with the PBC on (d) using $\bar{V}=V_{50}$. Here, $u_{\text {conv }}$ is equal to the horizontal velocity very far from the dihedral :

$$
u_{\text {conv }}=1 \text {. }
$$

At the begining of the simulation, the NRBC gives rise to a small modification of the horizontal velocity isolines distribution, and provides strong acoustic reflections coming from the outflow boundary, which can be seen on the pressure field, whereas the PBC does not (FIGURE 17).

During the simulation, the PBC does not change the solution. After a long time of simulation, the NRBC converges towards a steady solution, but with a recirculation area, and with the horizontal velocity isolines perpendicular to the outflow boundary (FIGURE 18), what is an unphysical behaviour.

¿From the solution obtained with the PBC at $R e_{\delta}=50$, we go on simulating the flow at $R e_{\delta}=100$, using $\bar{V}=V_{50}$ for the PBC implementation. At the begining of the simulation, 
no perturbation is detected on the outflow boundary and vortex dropping arises at point B. It corresponds to a transition regime, because of the change of $R e_{\delta}$. The vortex are then convected, and leave the computational domain. In order to illustrate this behaviour, the Jeong and Hussain criterion [40] as well as the pressure isolines are plotted at two different times (FIGURE 19). After a long time of simulation, an established regime can be analysed. the $\mathrm{PBC}$ provides a steady solution with a recirculation zone like obtained with the use of the buffer zone at the same $R e_{\delta}$. On the other hand, the NRBC provides an unsteady periodic solution (FIGURE 20), whose fundamental frequency depends on the $\mathrm{n}$ parameter, like proved by Hernandez in a similar configuration [25].

A last simulation is then performed at $R e_{\delta}=400$, using $\bar{V}=V_{100}$ computed above for the PBC implementation. Whatever the type of the BC used, the solution is unsteady, like obtained with the buffer zone at the same $R e_{\delta}$. We plot the pressure evolution in the domain as a function of the time at a given point in the flow (FIGURE 21). We can observe that the signal corresponding to the $\mathrm{PBC}$ is far closer to the signal obtained with the buffer zone than the signal corresponding to the NRBC. Moreover, the energy spectra of the signals show the presence of a low frequency for the NRBC solution, which is not present for the PBC solution and for the the solution obtained with the buffer zone. This low frequency is already present at $R e_{\delta}=100$, and corresponds to the travel of acoustic waves from the artificial boundary back to the corner. Finally, the pressure field obtained after a simulation time equal to 600 is displayed on FIGURE 22 . We see clearly that the PBC provides about the same solution despite a small delay than the solution obtained with the buffer zone. But the computation of the solution with the buffer zone requires roughly $50 \%$ more cpu time.

\section{CONCLUSION}

For subsonic compressible flows, specially when vortices cross the artificial frontiers of the computational domain, it is not relevant to cancel the amplitude of the entering characteristic waves. So, in this work the aim is to preserve these amplitudes in a stable way, which can not be done for instance by a simple extrapolation procedure. In order to do it, a reference flow as well as a convection velocity are used to take into account the flow behaviour outside of the domain of simulation. The results show the efficiency of this strategy as the computed flows in various configurations do not exhibit unphysical phenomena due to the presence of artificial boundary. 


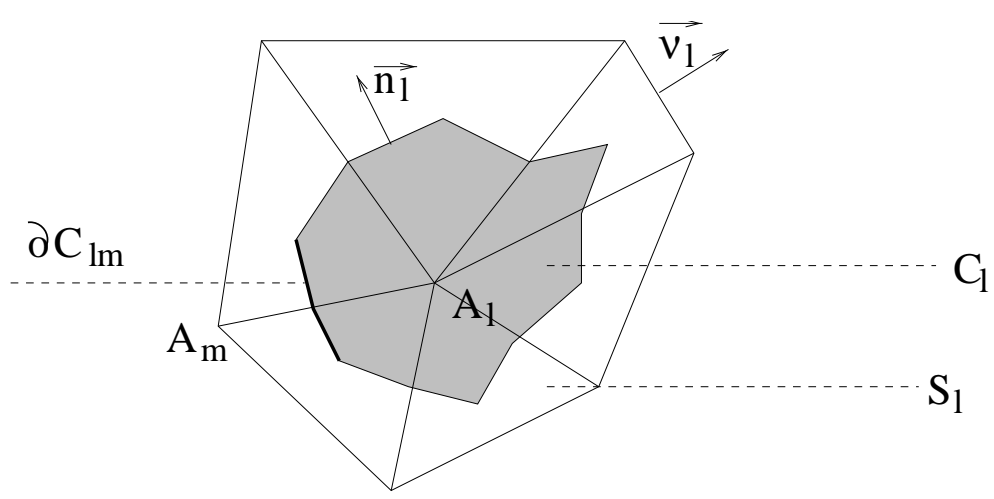

Figure 1: Control volumes $C_{l}$ and $S_{l}$ around $A_{l}$.

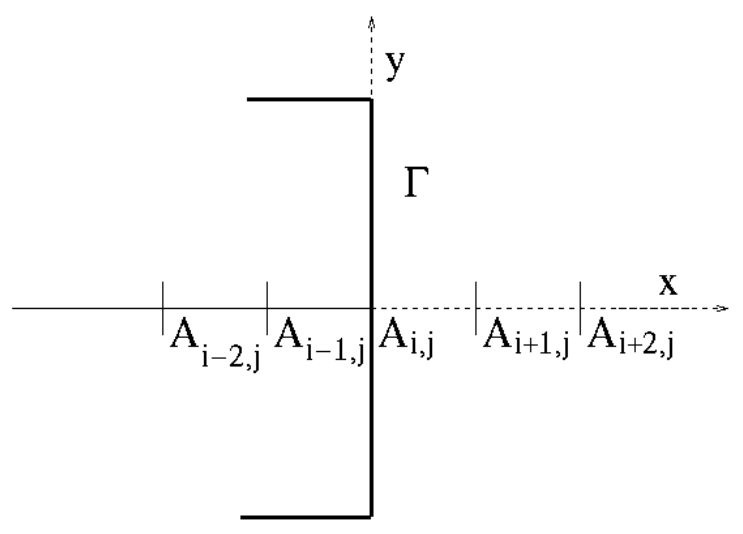

Figure 2: The $A_{i, j}$ point on $\Gamma$. 


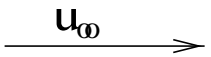

(c)

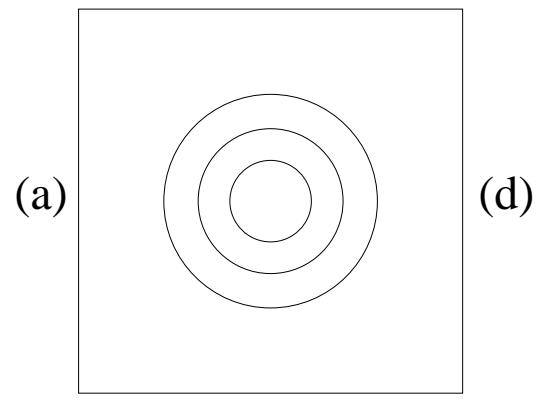

(b)

Figure 3: Computational domain. 


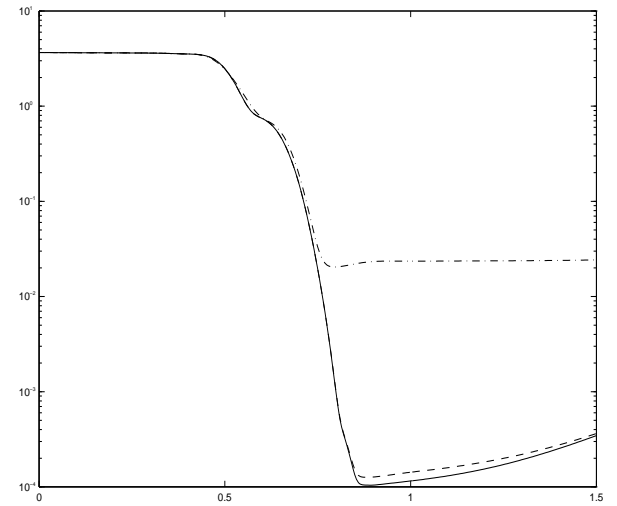

$\|\omega\|_{L_{2}}=f(t)$

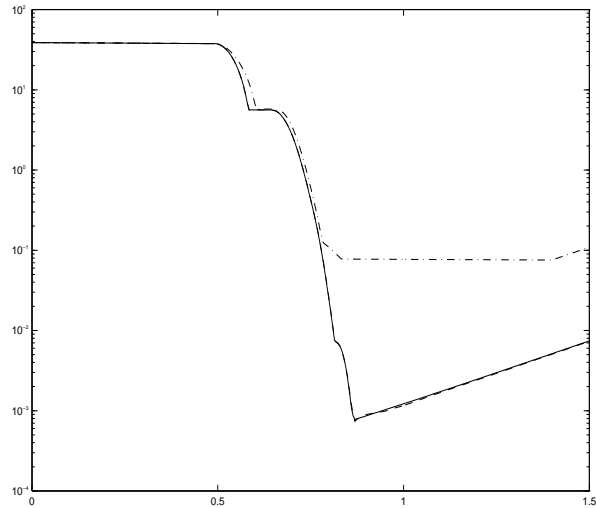

$\|\omega\|_{L_{\infty}}=f(t)$

Vortex $V_{1}$

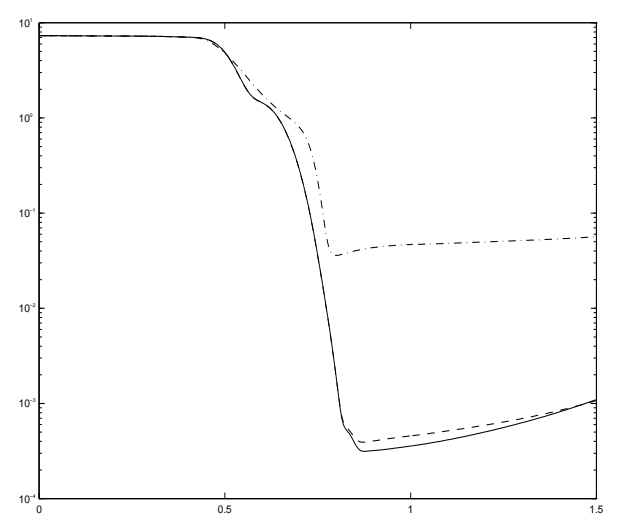

$\|\omega\|_{L_{2}}=f(t)$

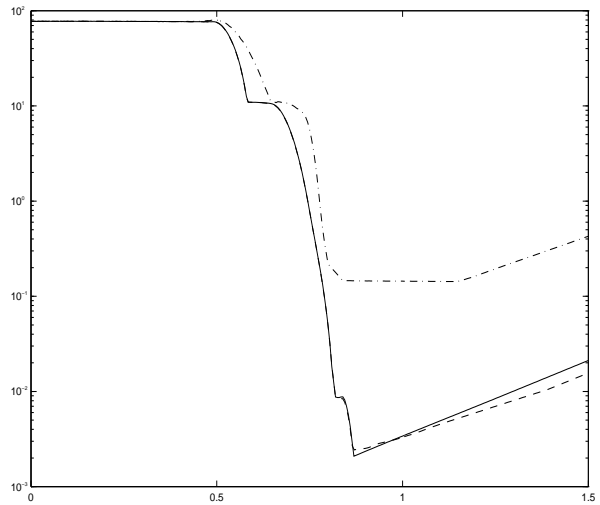

$\|\omega\|_{L_{\infty}}=f(t)$

vortex $V_{2}$

Figure 4: Vorticity evacuation.

Comparison of the NRBC solution (-.-.-.) and the PBC solution (- - -) to the RS (plain line). 


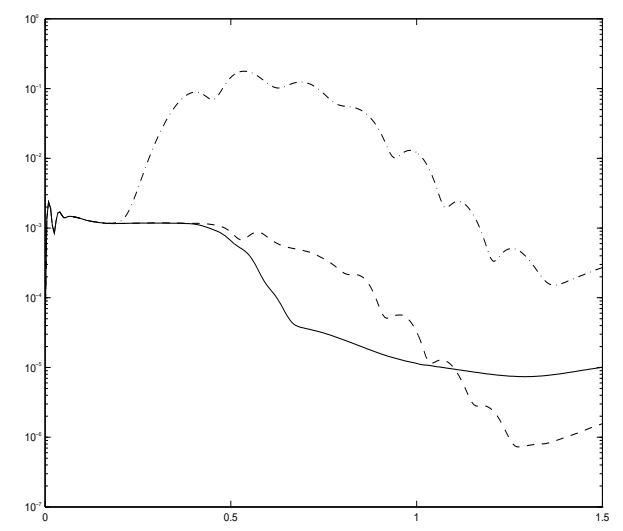

Vortex $V_{1}, \quad r=f(t)$

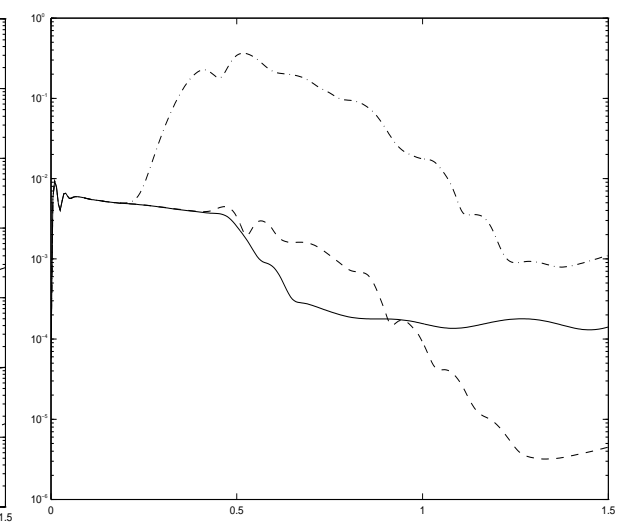

Vortex $V_{2}, \quad r=f(t)$

Figure 5: Reflection coefficients.

Comparison of the NRBC solution (-.-.-.) and the PBC solution (- - -) to the RS (plain line).
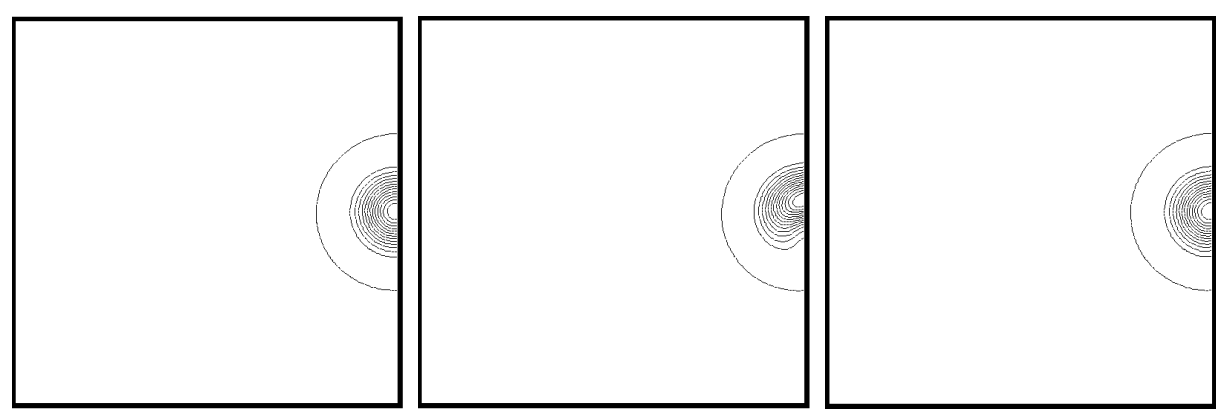

$t=0.5$, vorticity isolines
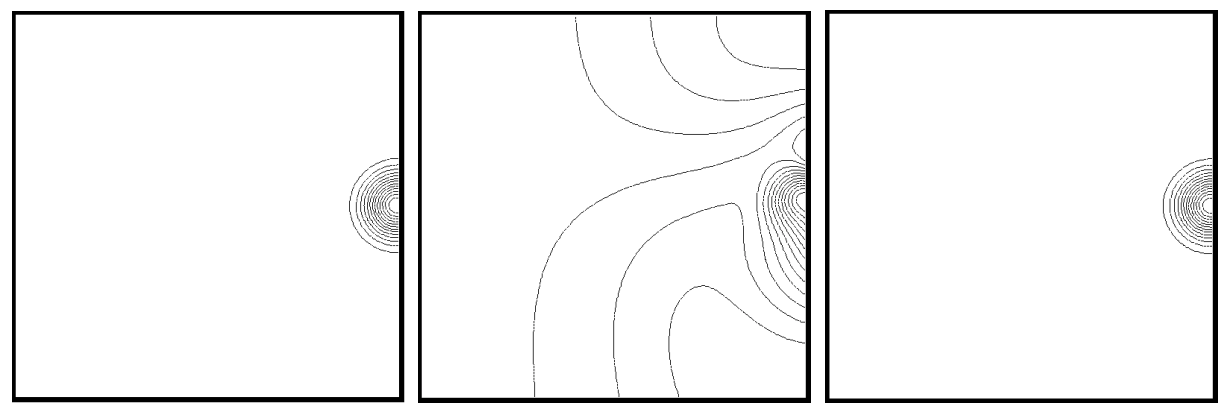

$t=0.5$, pressure isolines

Figure 6: $V_{1}$ vortex convection, vorticity and pressure fields.

Comparison of the NRBC solution (middle) and the PBC solution (right) to RS (left). 


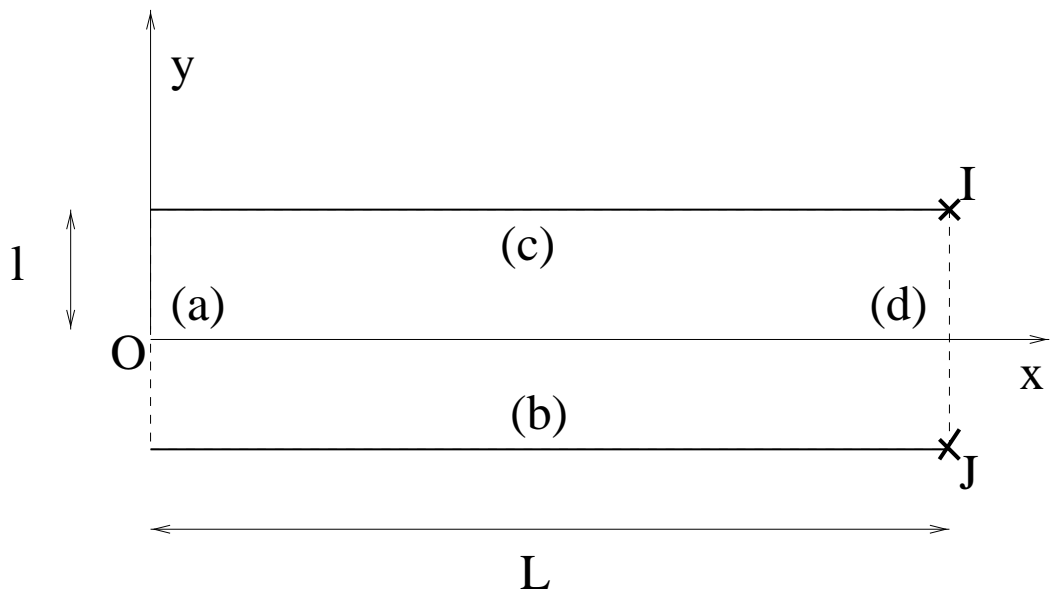

Figure 7: Poiseuille flow. 


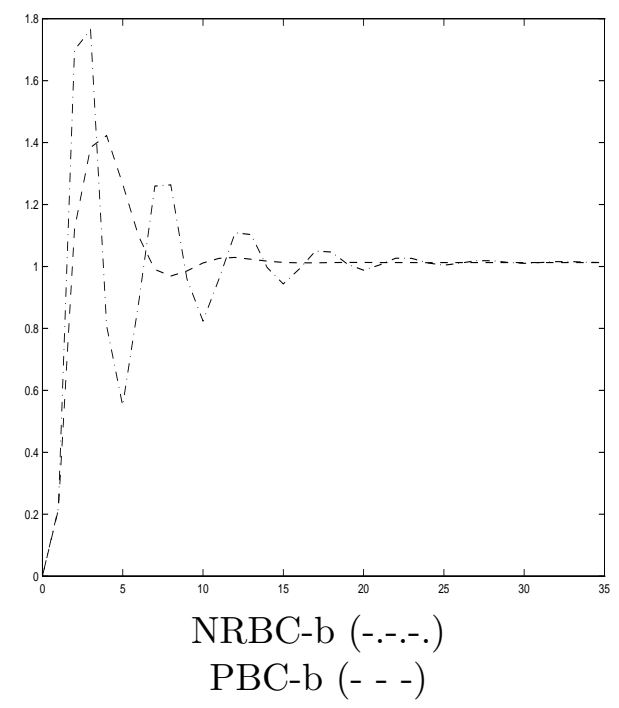

Figure 8: Mass flux through the outflow boundary.

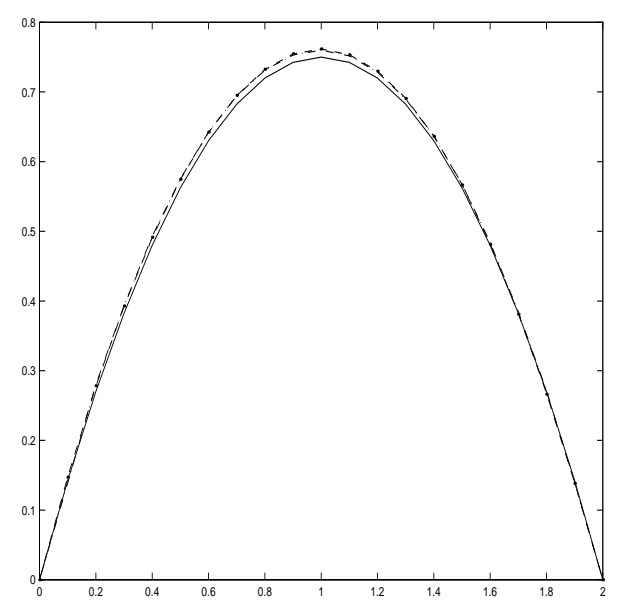

(a)

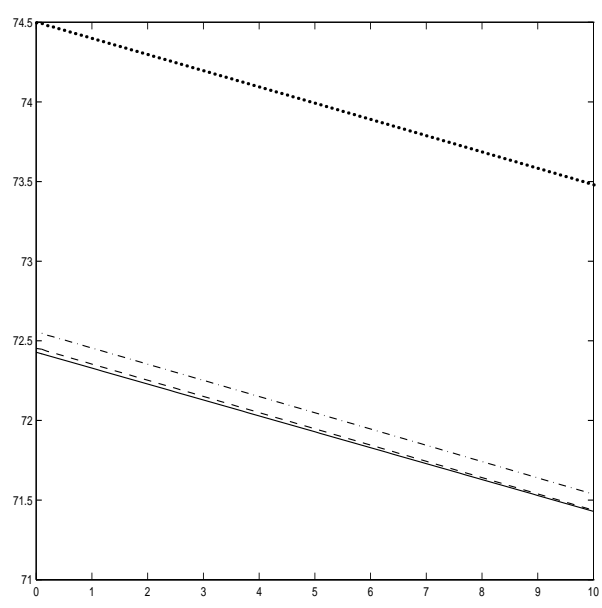

(b)

Figure 9: Steady state reached.

Comparisons between the PNRBC solution (......), the NRBC-b solution (-.-.-.), and the PBC-b solution (- - -), to the exact solution (plain). 


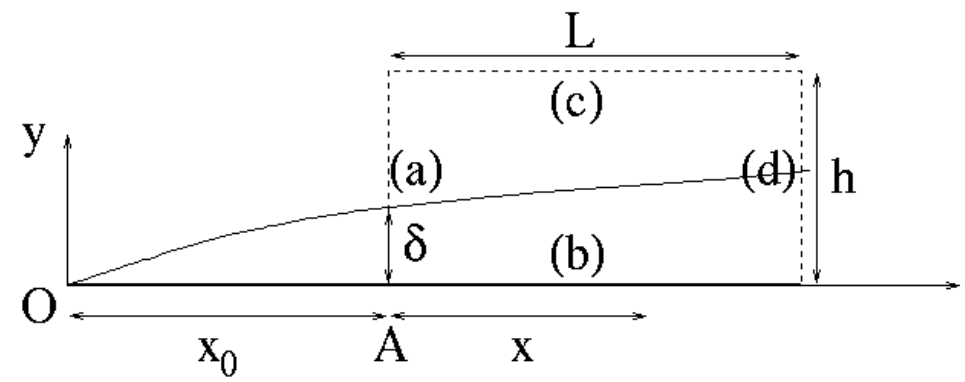

Figure 10: Boundary layer. 


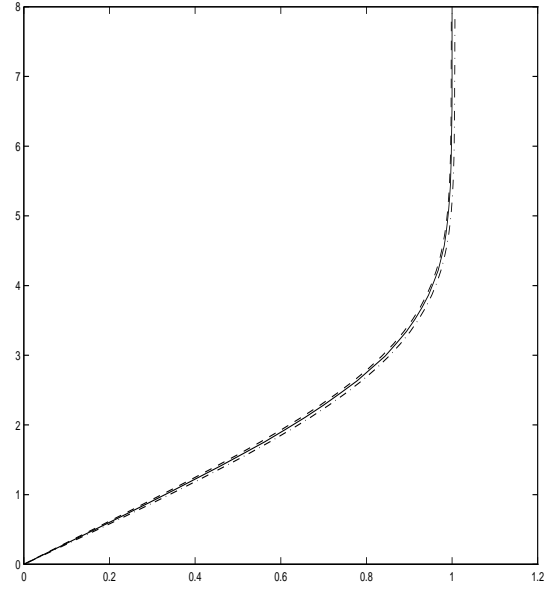

middle transverse section

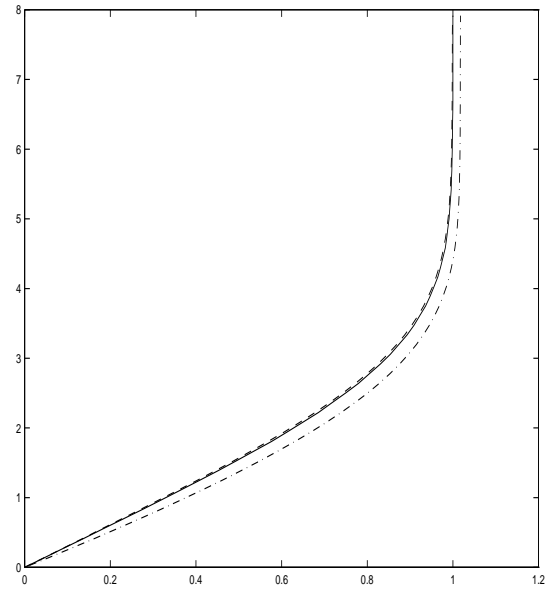

outflow transverse section

Horizontal velocity

$\eta=f\left(u_{\text {num }}\right)$

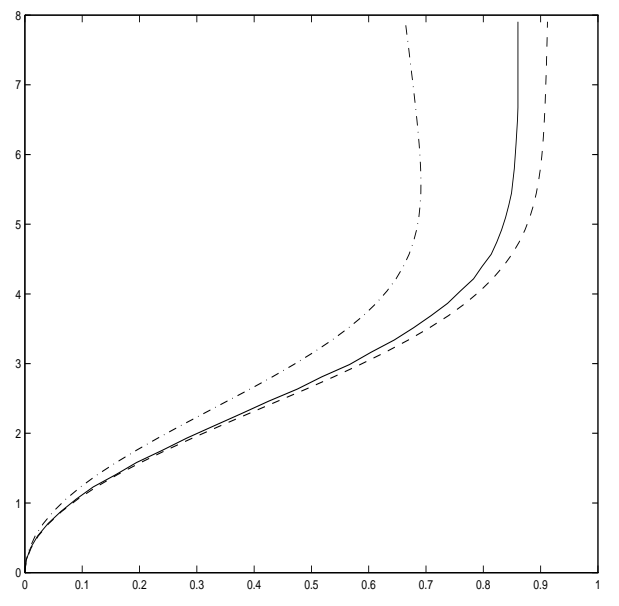

middle transverse section

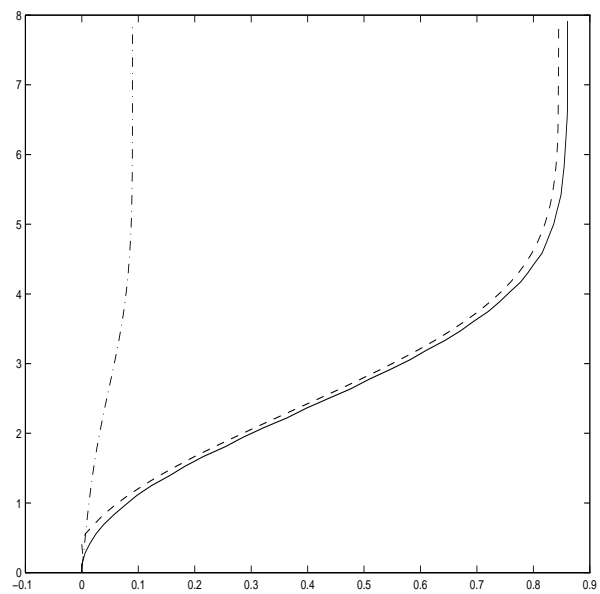

outflow transverse section

Vertical velocity

$$
\eta=f\left(v_{\text {num }}\right)
$$

Figure 11: Boundary layer .

Comparison of the NRBC solution (-.-.-.) and the PBC solution (- - -)

to the Blasius solution (plain line). 

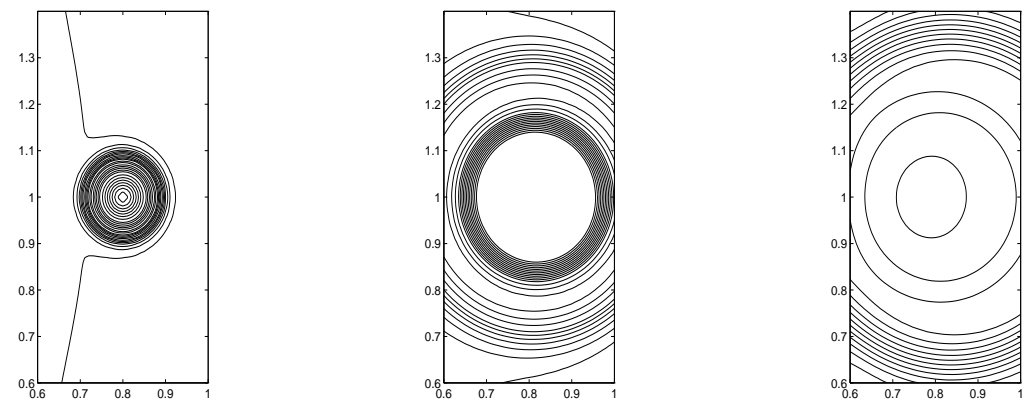

$t=0.00$

$t=0.02$

$t=0.04$

Reference Solution
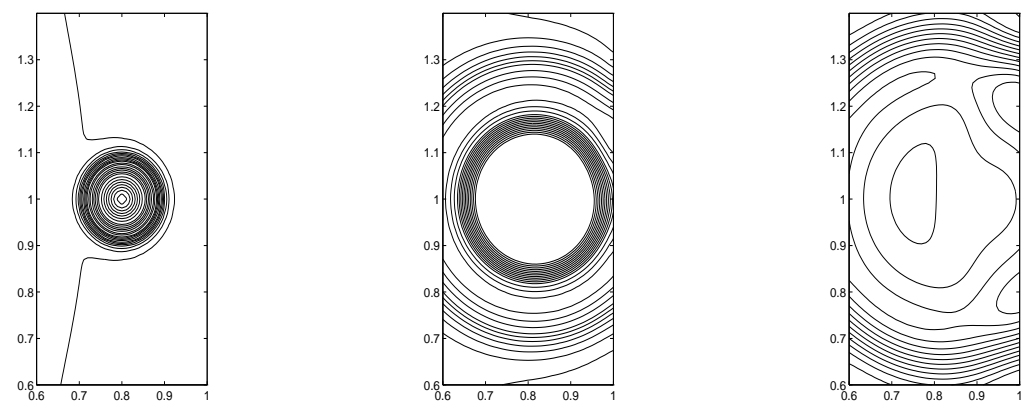

$t=0.00$

$t=0.02$

NRBC
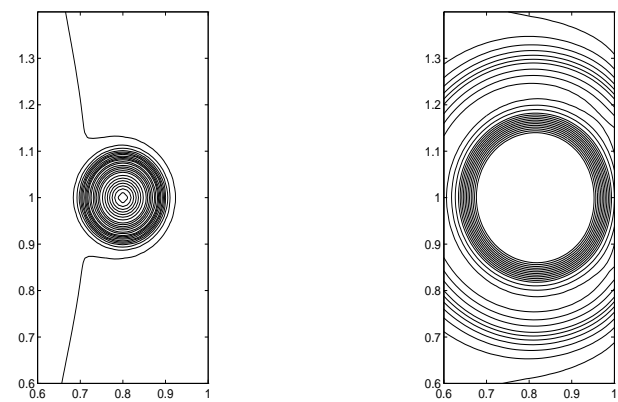

$t=0.00$

$$
t=0.02
$$

PBC, $u_{\text {conv }}=\frac{2 u_{0}}{3}$

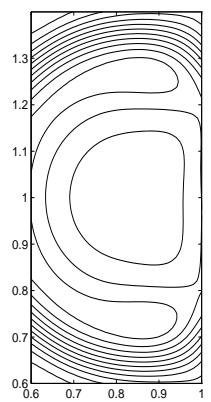

$t=0.04$
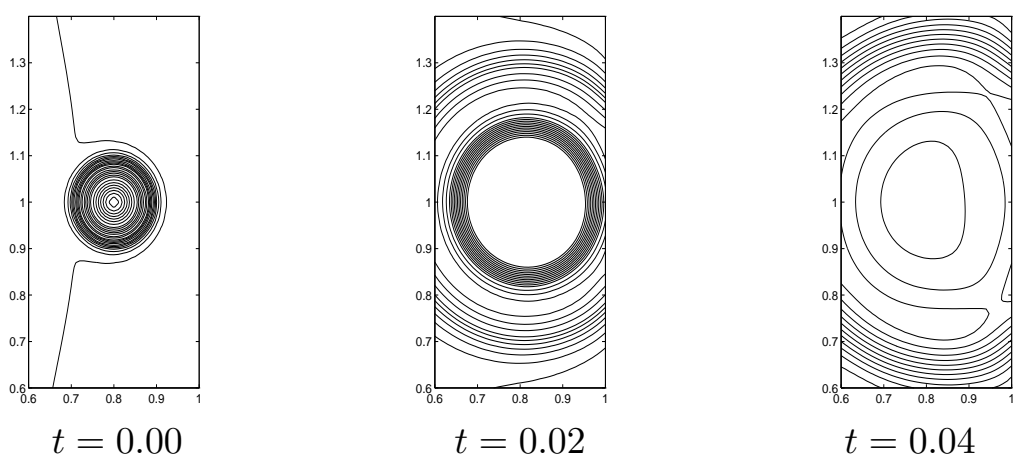

$$
\begin{aligned}
t & =0.02 \\
\text { PBC, } u_{\text {conv }} & =\frac{2 u_{0}}{3}\left(1+\frac{1}{M}\right)
\end{aligned}
$$$$
t=0.04
$$

Figure 12: Pressure isolines evolution for the acoustic perturbation.

Comparison of the NRBC solution (middle) and the PBC solution (bottom) to the RS (top). 


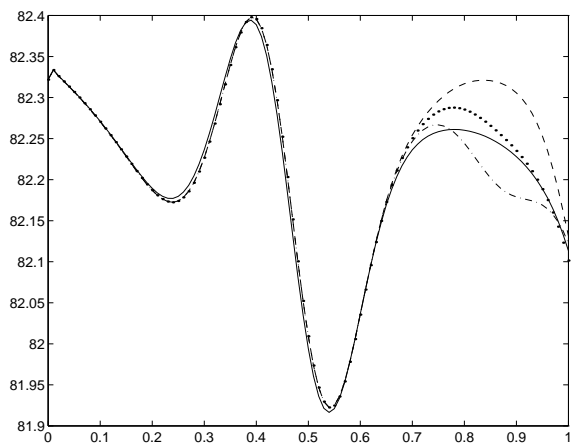

Figure 13: Longitudinal pressure evolution along $y=1$ at time $t=0.04$. Comparison of the NRBC solution (-.-.-.), the PBC solution with $u_{\text {conv }}=\frac{2 u_{0}}{3}(---)$ and the PBC solution with $u_{\text {conv }}=\frac{2 u_{0}}{3}\left(1+\frac{1}{M}\right)(\ldots)$ to the reference solution (plain line).

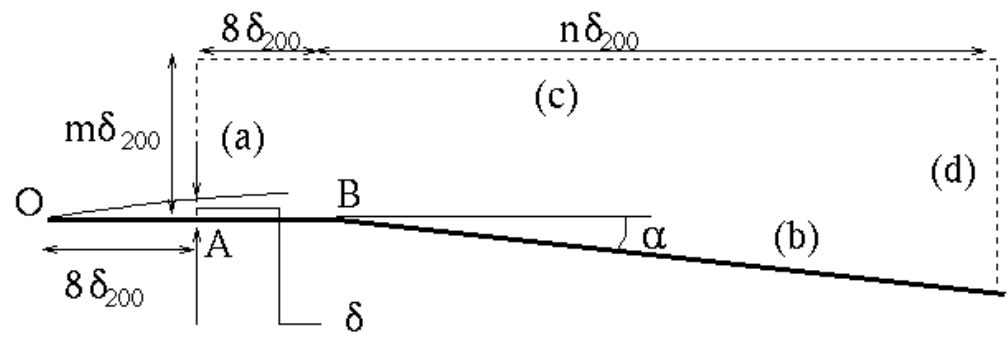

Figure 14: Dihedral plate configuration.

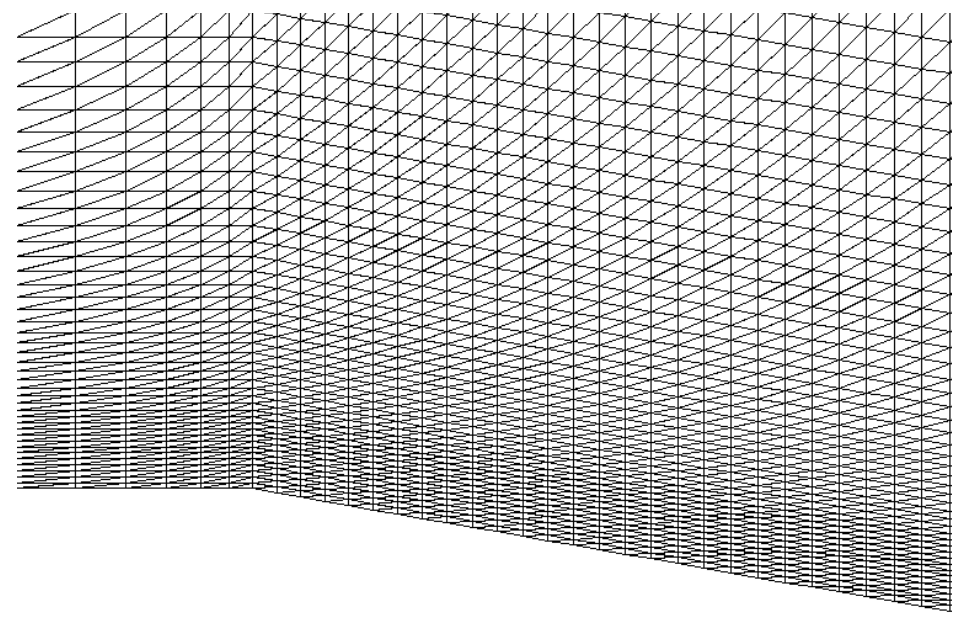

Figure 15: Mesh of the computational domain around point B. 


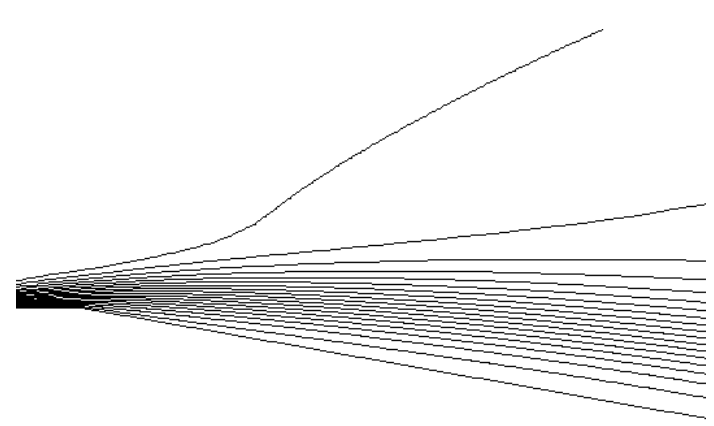

Horizontal velocity isolines

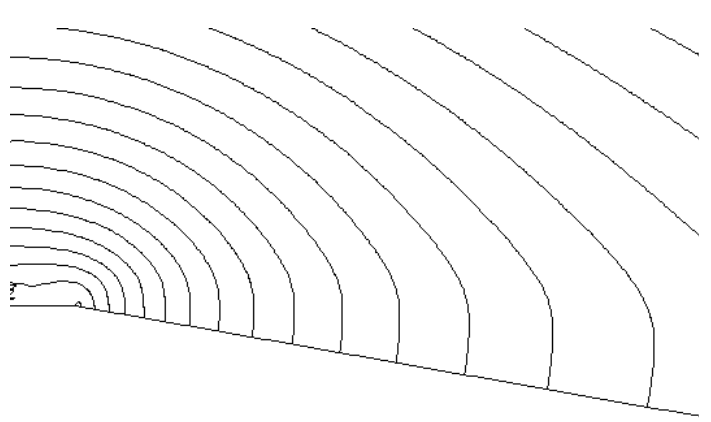

Pressure isolines

Figure 16: $R e_{\delta}=50$, use of a buffer zone. 


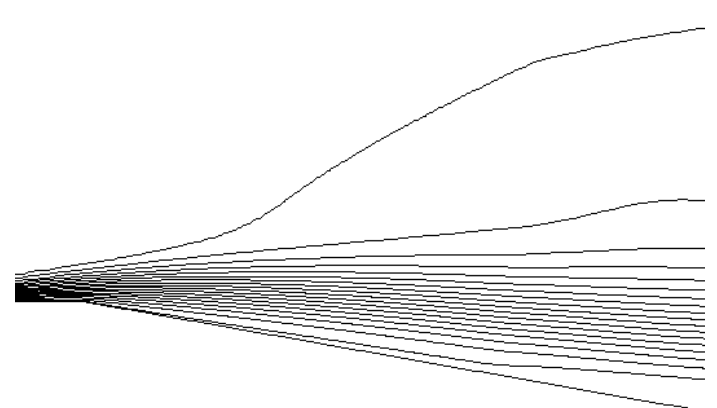

Horizontal velocity field, NRBC

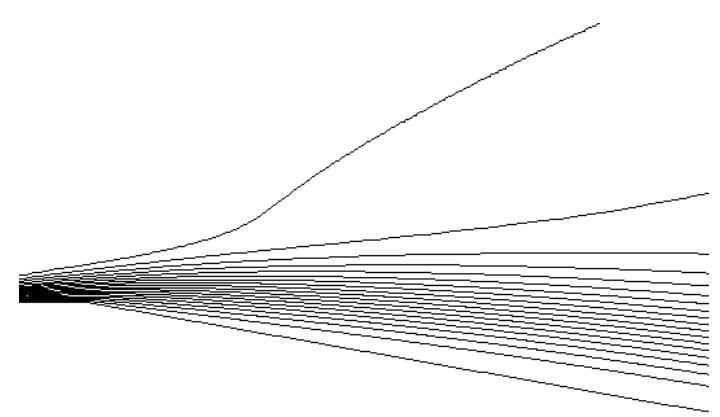

Horizontal velocity field, PBC

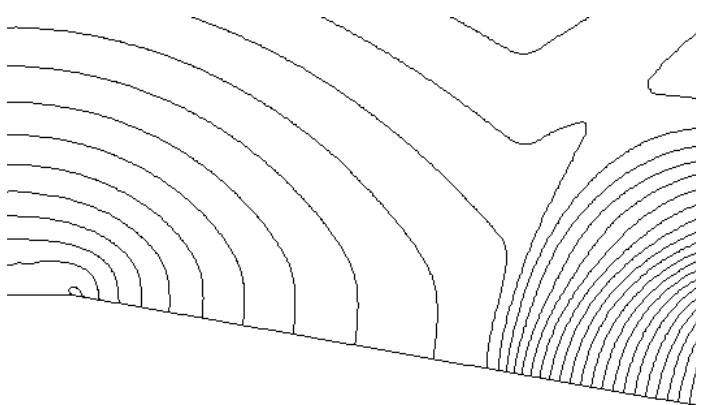

Pressure field, NRBC

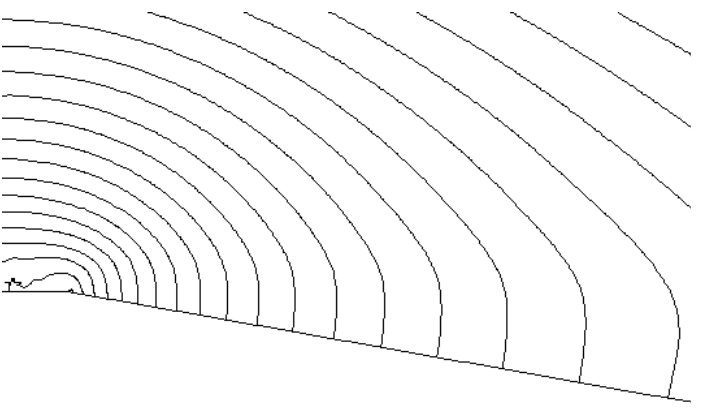

Pressure field, PBC

Figure 17: $R e_{\delta}=50$, comparison between the NRBC (top) and the PBC (bottom). Begining of the simulation.

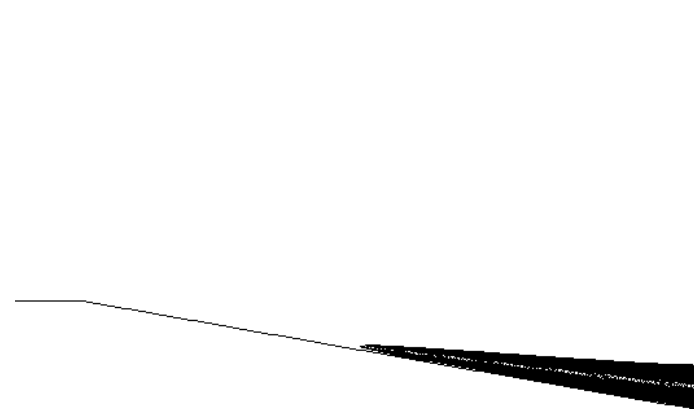

Negative horizontal velocities

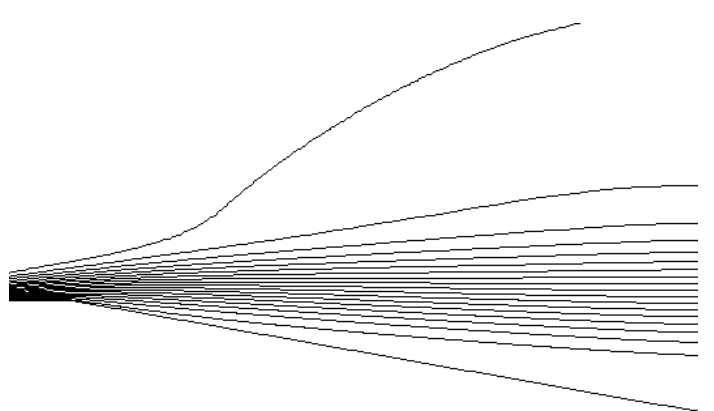

Horizontal velocity isolines

Figure 18: $R e_{\delta}=50$, NRBC.

Steady state reached. 


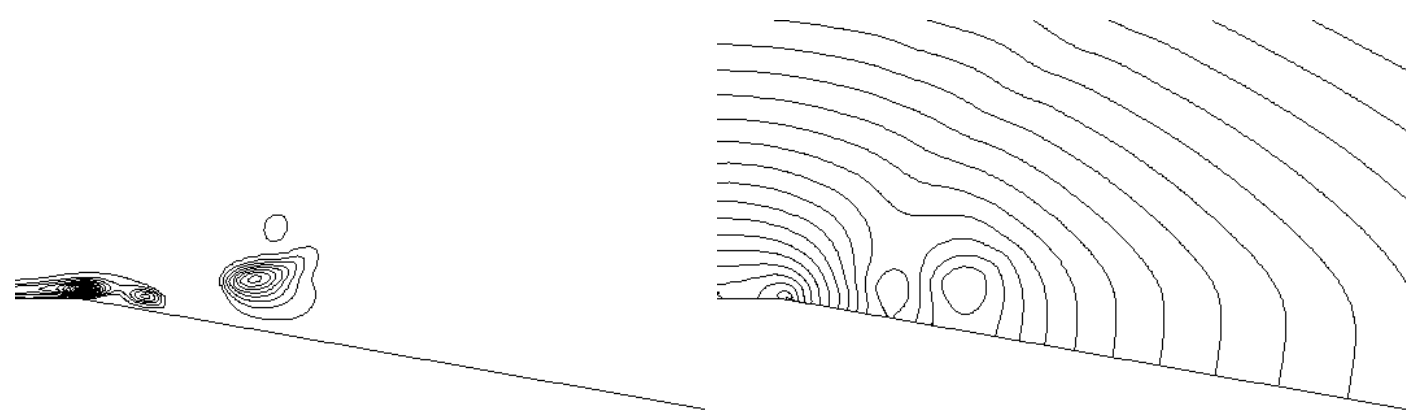

$$
t_{0}+30 \quad t_{0}+30
$$

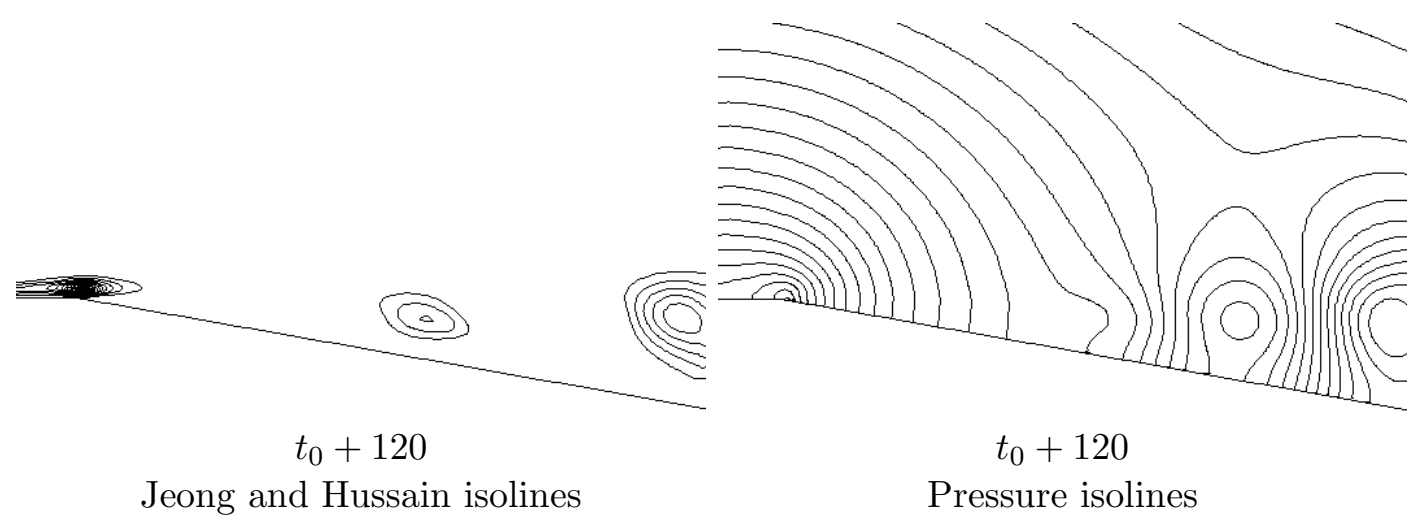

Figure 19: $\operatorname{Re}_{\delta}=100$, Begining of the simulation .

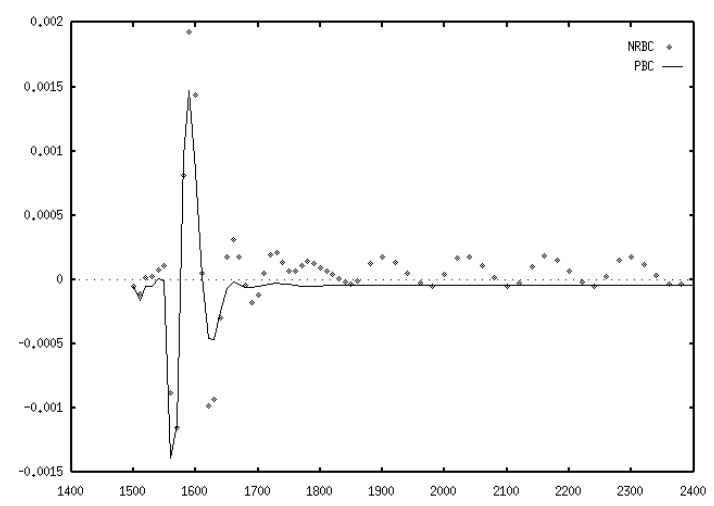

Figure 20: $R e_{\delta}=100$, established regime.

Temporal evolution of the J. and H. criterion at a given point in the flow. Comparison of the NRBC solution (plotted line) to the PBC solution (plain line). 

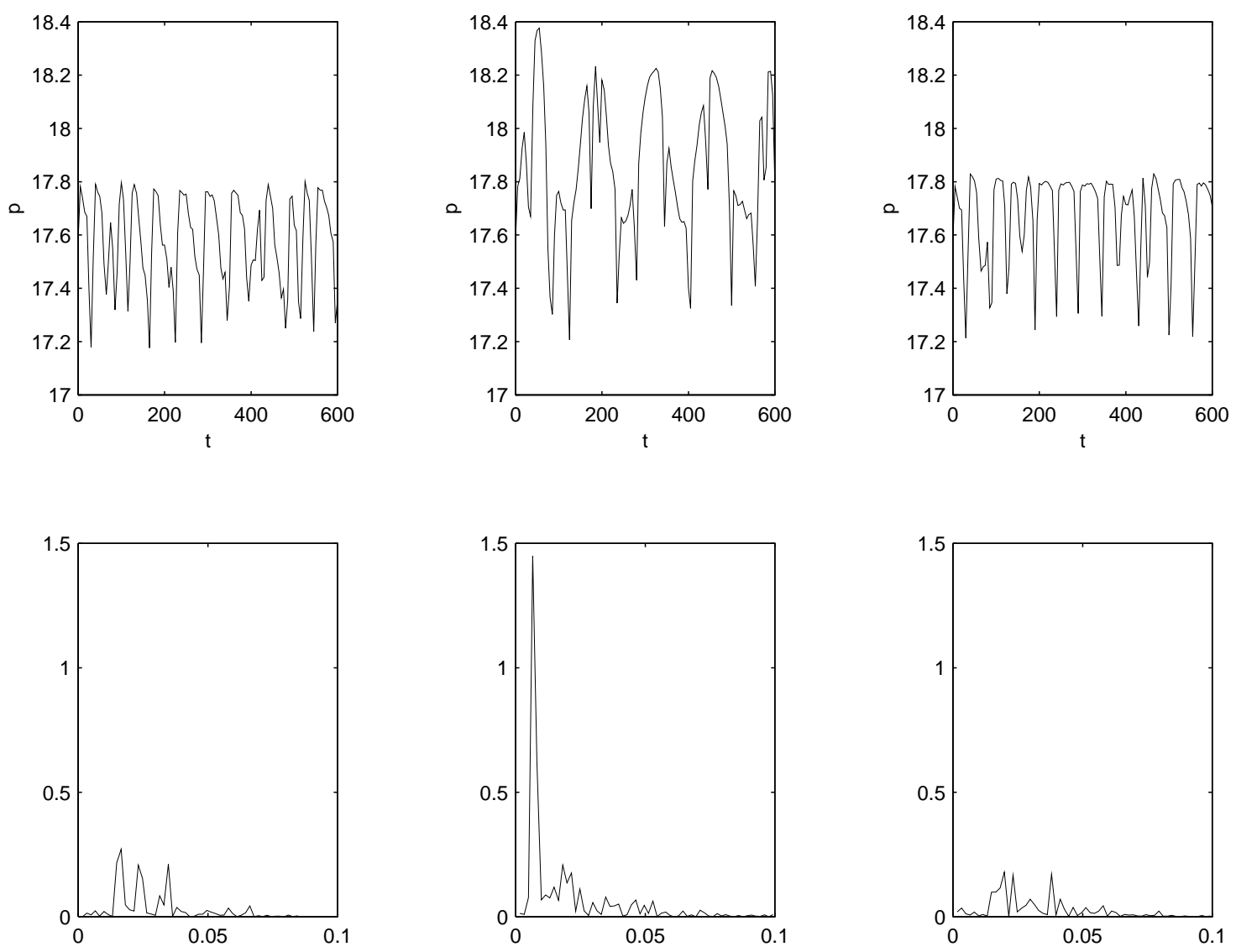

Figure 21: $R e_{\delta}=400$.

Temporal evolution of the pressure at a given point in the flow (top) and energy spectra of the signals (bottom).

Comparison of the NRBC solution (middle) and the PBC solution (right) to the buffer solution (left). 

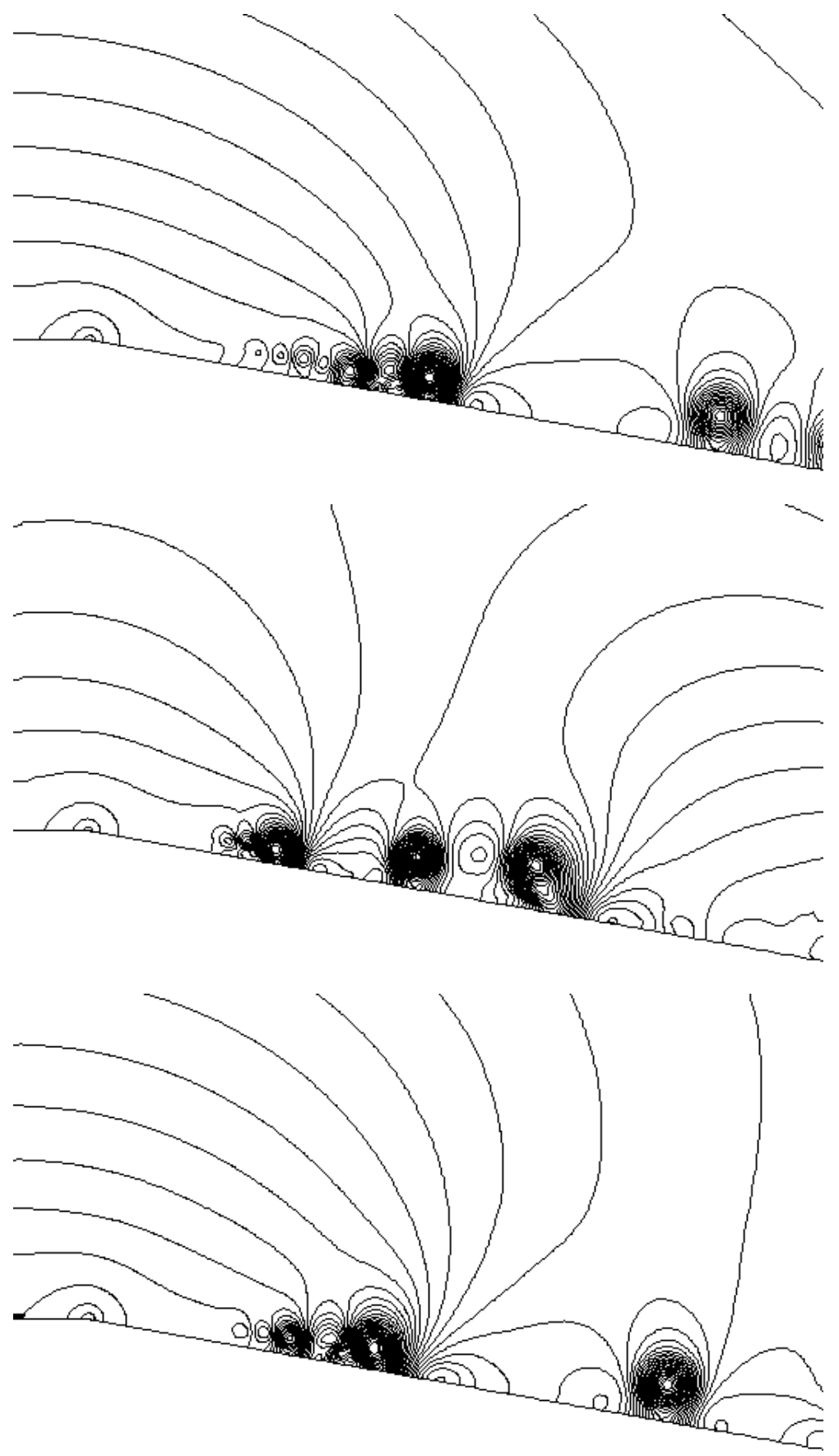

Figure 22: $R e_{\delta}=400$, pressure field at time $t=600$.

Comparison of the NRBC solution (middle) and the PBC solution (bottom) to the buffer solution (top). 


\section{REFERENCES}

\section{References}

[1] H.O. Kreiss, 'Initial boundary value problems for hyperbolic systems', Communication on Pure and Applied Mathematics, 23, 277-298 (1970).

[2] J.C. Strikwerda, 'Initial boundary value problems for incompletely parabolic systems', Communication on Pure and Applied Mathematics, 30, 797-822 (1977).

[3] R.L. Higdon, 'Initial boundary value problems for linear hyperbolic systems', SIAM review, 28(2), 177-217 (1986).

[4] B. Gustaffson and A. Sundstrom, 'Incompletely parabolic problem in fluid dynamics', SIAM journal of Applied Mathematics, 35(2), 343-357 (1978).

[5] J. Oliger and A. Sundstrom, 'Theoritical and practical aspects of some initial boundary value problems in fluid dynamics', SIAM Journal of Applied Mathematics, 35(3), 419-446 (1978).

[6] P.Dutt, 'Stable boundary conditions and difference schemes for Navier-Stokes equations', SIAM Journal of Numerical Analysis, 25(2), 245-267 (1988).

[7] J.S. Hesthaven and D. Gottlieb, 'A stable penalty method for the compressible NavierStokes equations - 1) open boundary conditions', SIAM Journal of Scientific Computation, 3, 579-612 (1996).

[8] D.H. Rudy and J.C. Strikwerda, 'A nonreflecting outflow boundary condition for subsonic Navier-Stokes calculations', Journal of Computational Physics, 36, 55-70 (1980).

[9] G.W. Hedstrom, 'Non reflecting boundary conditions for non linear hyperbolic systems', Journal of Computational Physics, 30, 222-237 (1979).

[10] K.W. Thompson, 'Time dependant boundary conditions for hyperbolic systems', Journal of Computational Physics, 68(1) (1987).

[11] B. Enquist and A. Majda, 'Absorbing boundary conditions for the numerical simulation of waves', Mathematics of Computation, 31(139), 629-651 (1977).

[12] H. Bayliss and E. Turkel, 'Far field boundary conditions for compressible flows', Journal of Computational Physics, 48, 182-199 (1982).

[13] L. Tourette, 'Artificial boundary conditions for the linearized compressible Navier-Stokes equations', Journal of Computational Physics, 137, 1-37 (1997).

[14] L. Tourette, 'Artificial boundary conditions for the linearized compressible Navier-Stokes equations, the discrete approach', Journal of Computational Physics, 144, 151-179 (1998).

[15] L. Halpern, 'Artificial boundary conditions for the linear advection diffusion equation', Mathematics of Computation, 46(174), 425-438 (1986).

[16] L. Halpern, 'Artificial boundary conditions for incompletely parabolic perturbations of hyperbolic systems', SIAM Journal of Mathematical Analysis, 22(5), 1256 (1991).

[17] K.W. Thompson, 'Time dependant boundary conditions for hyperbolic systems - Part 2', Journal of Computational Physics, 89, 439-461 (1990).

[18] D.H. Rudy and J.C Strikwerda, 'Boundary conditions for subsonic compressible NavierStokes calculations', Computers and Fluids, 9, 327-338 (1981).

[19] T.J. Poinsot and S.K. Lele, 'Boundary conditions for direct simulations of compressible viscous flows', Journal of Computational Physics, 101, 104-129 (1992).

[20] M. Baum, T. Poinsot and D. Thevenin, 'Accurate boundary conditions for multicomponent reactive flows', Journal of Computational Physics, 116, 247-261 (1994). 
[21] M.B. Giles, 'Non reflecting boundary conditions for Euler equation calculations', AIAA Journal, 28(12), 2050-2058 (1990).

[22] F. Nicoud, 'On the amplitude of waves in characteristic boundary conditions for Euler equations', Technical report, CERFACS (1998).

[23] T. Colonius, S.K.Lele and M. Parviz, 'Boundary conditions for direct computation of aerodynamic sound generation', AIAA journal, 31(9), 1574-1582 (1993).

[24] F.F. Grinstein, 'Open boundary conditions in the simulation of subsonic turbulent shear flows', Journal of Computational Physics, 115, 43-55 (1996).

[25] G. Hernandez, 'Controle actif des instabilites hydrodynamiques des ecoulements subsoniques compressibles', PhD Thesis, CERFACS (1996).

[26] C.L. Street and M.G. Macaraeg, 'Spectral multi-domain for large-scale fluid dynamics simulations', Applied Numerical Mathematics, 5, 123-139 (1990).

[27] Z. Liu and Z. Liu, 'Fourth order finite difference and multigrid method for modeling instabilities in flat plate boundary layer - 2D and 3D approaches', Computers and Fluids, 23(7), 955-982 (1994).

[28] Pruett, Zang, Chang and Carpenter, 'Spatial direct numerical simulation of high-speed boundary layers flows. Part1: Algorithmic considerations and validation', Theoritical and Computational Fluid Dynamics, 7, 49-76 (1995).

[29] B. Wasistho, B.J. Geurts and J.G.M. Kuerten, 'Simulation techniques for spatially evolving instabilities in compressible flows over a flat plate', Computers and Fluids, 26(7), 713-739 (1997).

[30] J.P. Berenger, 'A perfectly matched layer for the absorption of electromagnetic waves', Journal of Computational Physics, 114, 185-200 (1994).

[31] F.Q.Hu, 'On absorbing boundary conditions for linearized Euler equations by a perfectly matched layer', Journal of Computational Physics, 129, 201-219 (1996).

[32] C.K.W. Tam, L. Auriault, and F. Cambuli, 'Perfectly matched layer as an absorbing boundary condition for the linearized Euler equations in open and ducted domains', Journal of Computational Physics, 144, 213-234 (1998).

[33] J.S. Hesthaven, 'On the analysis and construction of perfectly matched layers for the linearized Euler equations', Journal of Computational Physics, 142, 129-147 (1998).

[34] C. Hirsch, 'Numerical computation of internal and external flows, John Wiley and sons, 2, (1995).

[35] A.Durand, 'Modélisation des équations de l'hydrodynamique moteur sur maillage non structuré hybride', Ph-D Thesis, DIMA-IFP (1996).

[36] L. Fezoui, S. Lanteri, B. Larrouturou and C. Olivier, 'Résolution numérique des équations de Navier-Stokes pour un fluide compressible en maillage triangulaire', Rapport de recherche INRIA 1033, (1989).

[37] C.H. Bruneau and P. Fabrie, 'Effective downstream boundary conditions for incompressible Navier-Stokes equations, International Journal for Numerical Methods in Fluids, 19, 693705 (1994).

[38] C.H. Bruneau and P. Fabrie, 'New efficient boundary conditions for incompressible NavierStokes equations : A well-posedness result', Mathematical Modelling and Numerical Analysis, 30(7), 815-840 (1996).

[39] J. Cousteix, 'Couche limite laminaire', Cepadues editions (1988).

[40] J. Jeong and F. Hussain, 'On the identification of a vortex', Journal of Fluid Mechanics, 285, 69-94 (1995). 